\title{
The EFSUMB Guidelines and Recommendations on the Clinical Practice of Contrast Enhanced Ultrasound (CEUS): Update 2011 on non-hepatic applications
}

Authors

Affiliations
F. Piscaglia' , C. Nolsøe², C. F. Dietrich ${ }^{3}$, D. O. Cosgrove ${ }^{4}$, O. H. Gilja ${ }^{5}$, M. Bachmann Nielsen ${ }^{6}$, T. Albrecht ${ }^{7}$, L. Barozzi ${ }^{8}$, M. Bertolotto ${ }^{9}$, O. Catalano ${ }^{10}$, M. Claudon ${ }^{11}$, D. A. Clevert ${ }^{12}$, J. M. Correas ${ }^{13}$, M. D’Onofrio ${ }^{14}$, F. M. Drudi ${ }^{15}$, J. Eyding ${ }^{16}$, M. Giovannini ${ }^{17}$, M. Hocke ${ }^{18}$, A. Ignee ${ }^{19}$, E. M. Jung ${ }^{20}$, A. S. Klauser ${ }^{21}$, N. Lassau ${ }^{22}$, E. Leen ${ }^{23}$, G. Mathis ${ }^{24}$, A. Saftoiu ${ }^{25}$, G. Seide ${ }^{26}$, P. S. Sidhu' ${ }^{27}$, G. ter. $\operatorname{Haar}^{28}$, D. Timmerman ${ }^{29}$, H. P. Weskott ${ }^{30}$

Affiliation addresses are listed at the end of the article.
Bibliography

DOI http://dx.doi.org/10.1055/

s-0031-1281676

Published online

August 26, 2011

Ultraschall in Med 2012; 33:

33-59 ๔ Georg Thieme Verlag

KG Stuttgart · New York .

ISSN 0172-4614

\section{Correspondence}

\section{Fabio Piscaglia MD, PhD}

Div. of Internal Medicine, General and University Hospital

S. Orsola-Malpighi

Via Albertoni 15

40138 Bologna, Italy

Tel.: + 39/0 51/63625 42/68

Fax: + 39/0 51/6362725

fabio.piscaglia@unibo.it

\section{Thematic sections}

$\nabla$

\begin{tabular}{|c|c|c|}
\hline & Thematic Section & Chairperson \\
\hline 1 & Introduction & $\begin{array}{l}\text { F. Piscaglia - } \\
\text { C. Nolsøe }\end{array}$ \\
\hline 2 & Generalities & D. Cosgrove \\
\hline 3 & Equipment & H.P. Weskott \\
\hline 4 & Investigator's training & O. H. Gilja \\
\hline 5 & Terminology & F. Piscaglia \\
\hline 6 & Safety & G. ter Haar \\
\hline 7 & Paediatric & C. Nolsøe \\
\hline 8 & Pancreas & M. D’Onofrio \\
\hline 9 & Endoscopic CEUS & C. F. Dietrich \\
\hline 10 & Gastrointestinal tract & O. H. Gilja \\
\hline 11 & Spleen & O. Catalano \\
\hline 12 & Kidney & M. Bertolotto \\
\hline 13 & Vesicoureteral reflux & M. Bertolotto \\
\hline 14 & Scrotum & P. S. Sidhu \\
\hline 15 & Abdominal Trauma & O. Catalano \\
\hline 16 & Lung and pleural lesions & G. Mathis \\
\hline 17 & Vascular & D. Clevert \\
\hline 18 & Cerebral vessels & G. Seidel \\
\hline 19 & Inflammatory joint diseases & A. S. Klauser \\
\hline 20 & Intracavitary & A. Ignee \\
\hline 21 & Lymphnodes & M. Hocke \\
\hline 22 & Tumour response assessment & E. Leen \\
\hline 23 & Breast & D. Cosgrove \\
\hline 24 & Adrenals & H.P. Weskott \\
\hline 25 & $\begin{array}{l}\text { Emerging CEUS applications } \\
\text { and future perspectives }\end{array}$ & \\
\hline A & Gynecology & D. Timmerman \\
\hline B & Perineum & C. F. Dietrich \\
\hline C & Urinary Bladder & F. M. Drudi \\
\hline D & Transplanted Kidney & M. Bertolotto \\
\hline E & Prostate Cancer & J. M. Correas \\
\hline $\mathrm{F}$ & Aorto-caval fistula & D. Clevert \\
\hline G & Free Tissue Transplants & E. M. Jung \\
\hline $\mathrm{H}$ & Extrahepatic Biliary System & C. F. Dietrich \\
\hline 26 & $\begin{array}{l}\text { Use of CEUS in patients with } \\
\text { renal failure }\end{array}$ & $\begin{array}{l}\text { J. M. Correas - } \\
\text { T. Albrecht }\end{array}$ \\
\hline 27 & Technical Appendix & $\begin{array}{l}\text { F. Piscaglia - } \\
\text { C. Nolsøe }\end{array}$ \\
\hline
\end{tabular}

\section{List of Abbreviations}

$\nabla$

AAA $=$ Abdominal Aortic Aneurysm

AUC $=$ Area Under the Curve

$\mathrm{CE}=$ Contrast Enhanced

CECT $=$ Contrast Enhanced Computed Tomo-

graphy

CEMRI $=$ Contrast Enhanced Magnetic Resonance

Imaging

CEUS $=$ Contrast Enhanced Ultrasound

CE-TCCS $=$ Contrast Enhanced Transcranial

Colour-Coded duplex Sonography

CE-EUS $=$ Contrast Enhanced Endoscopic

Ultrasound

$\mathrm{CT}=$ Computed Tomography

DCE-US = Dynamic Contrast Enhanced Ultra-

sound

EFSUMB = European Federation of Societies for

Ultrasound in Medicine and Biology

EUS $=$ Endoscopic Ultrasound

HCC = Hepatocellular Carcinoma

NFS = Nephrogenic systemic fibrosis

GIST $=$ Gastrointestinal Stromal Tumour

$\mathrm{MI}=$ Mechanical Index

RECIST $=$ Response Evaluation Criteria in Solid

Tumours

PET $=$ Positron Emission Tomography

$\mathrm{RI}=$ Resistance Index

$\mathrm{ROI}=$ Region of Interest

$\mathrm{TIC}=$ Time Intensity Curve

UCA $=$ Ultrasound Contrast Agent

US = Ultrasound

VUR $=$ Vesico-ureteral Reflux

WFUMB $=$ World Federation of Societies for

Ultrasound in Medicine and Biology 


\section{Introduction}

$\nabla$

The first studies on the clinical use of ultrasound contrast agents (UCAs) with contrast specific imaging techniques were published at the beginning of this century using Levovist ${ }^{\circledR}$ as UCA [1, 2]. A couple of years later sulphur hexafluoride (SonoVue $^{\circledR}$, Bracco, Milan) was marketed in Europe, opening the era of real time low mechanical index (MI) contrast enhanced ultrasound (CEUS). The European Federation of Societies for Ultrasound in Medicine and Biology (EFSUMB) released the first Guidelines on the use of CEUS in 2004 [3]. This document, which focused mainly on liver applications, contributed to the rapid expansion of CEUS and sanctioned the acronym "CEUS". CEUS was felt to be a revolutionary technique and many new applications, besides those in the liver, were developed in the following years. Therefore, in the update of the Clinical Recommendations on the use of CEUS by the EFSUMB, published in 2008 [4], applications in some other organs were described. Since then, there has been exponentially increasing interest in the clinical applications of CEUS and new fields have been investigated, so that nearly all organ systems have now been subjected to some kind of CEUS study. More than half of the approximately 900 original investigative articles (editorials and letters excluded) found in PubMed ${ }^{\circledR}$ under the search term "Contrast Enhanced Ultrasound" by the end of 2010 were published after 2008, after the last update of the EFSUMB guidelines [4]. Therefore, in 2010 EFSUMB felt it was the right time to start preparing a new update of the Guidelines. In the light of the expansion in CEUS usage, it was felt that a new update on CEUS for liver applications would be more beneficial to the clinical community if endorsed on a worldwide basis. Therefore, an agreement was reached with the World Federation of Societies for Ultrasound in Medicine and Biology (WFUMB) to prepare joint guidelines with EFSUMB exclusively for liver applications, taking advantage of its previous experience and of the previously published document [4]. These are expected to be issued as double simultaneous publications in Ultraschall in der Medizin/European Journal of Ultrasound and in Ultrasound in Medicine and Biology. At the same time the EFSUMB Executive Bureau decided that non-liver applications would be covered by a dedicated ESFUMB document under the guidance of a steering committee. The steering committee chose a panel of authors on the basis of their publication records in the various fields of interest and their reputation as international experts in research and teaching CEUS. By necessity, this list of co-authors could include only a limited number of persons and we acknowledge that many other highly qualified CEUS operators are active throughout Europe and have published valuable scientific articles on different CEUS topics. EFSUMB regrets not being able to include all of them in this process.

These Clinical Recommendations on the use of CEUS are based on comprehensive literature surveys including results from prospective clinical trials. In the case of topics for which no or little significant study data were available, evidence was obtained from expert committee reports or was based on the views of experts in the field during a consensus conference held in Frankfurt in April 2011.

As with the previous editions, these recommendations provide general advice for the use of UCAs. They are intended to create standard protocols for the use and administration of UCAs and improve the patient management. Individual cases must be managed on the basis of all clinical data available for that specific patient.

A few points are worth special mention. Many applications included in this article continue to be off-label. UCAs registered in Europe are licensed only for cardiac or, in the case of SonoVue $^{\circledR}$, for liver, breast and vascular applications. Levovist ${ }^{\circledR}$ is also registered for vesico-ureteric studies, but is practically no longer marketed, and is expected ultimately no longer be available. The current legal requirements for registration of pharmaceutical products in Europe are strict. In order for a new indication to be registered, the manufacturer must provide data on safety and efficacy, with a dedicated phase III trial specifically designed to obtain that registration approval. Diagnostic agents, including contrast microbubbles, are not exempt from this rule, which is designed to protect patients from misuse of drugs or diagnostic agents but, on some occasions, may limit the potential benefit to patients. In fact, applications for indications not only follow clinical or scientific needs, but also the financial expectations of the producer. Similarly, for some products, the marketing forecast for income from additional indications is lower than the cost of a registration trial, so that new indications, especially for some organs, are highly unlikely to appear. However, lack of registration does not mean lack of efficacy of a marketed compound. Having said that, we must not forget that UCAs continue to be offlabel for many non-liver indications detailed in the present recommendations. This means that patients should be informed and must consent to the investigation, and this is the responsibility of the operator. Indeed, non-liver CEUS is frequently performed on demand, to address specific questions raised in the individual patient. This explains (and justifies!) why it is not simple to write definitive guidelines and recommendations for the extrahepatic applications of CEUS.

CEUS has a number of distinct advantages over CT and MRI. It can be performed immediately, without any preliminary laboratory testing, and it can be carried out in a variety of scenarios (bedside, operating room, CT suite, etc.). Importantly also, it operates in real time so that rapid changes can be captured.

What then is the role of the current document? Firstly, it explains rationale for proceeding with the investigation, both to patients and clinicians, since for most situations the benefits are clearly outlined in this article. Secondly, it reports on the extremely rare and limited risks to which patients are exposed when they undergo a CEUS examination. These two points together facilitate and expedite the explanation of the risk/benefit ratio for the specific investigation to which a patient may be asked to consent. One example of this is in paediatric applications, for which the EFSUMB officers have already expressed a similar point of view [5].

Finally, based on the literature and the experts' consensus, an attempt was made to provide a recommendation level for each indication. The British guidelines for reporting on this topic have been used, as found at http://www.essentialevidenceplus.com/product/ebm_loe.cfm?show = guidelines, accessed in March 2011, in which the more detailed criteria of the Centre for Evidence Based Medicine in Oxford have been incorporated after slight adaptation ( Table $\mathbf{1}$ ).

A: There is good research-based evidence to support the recommendation.

$\mathrm{B}$ : There is fair research-based evidence to support the recommendation. 


\begin{tabular}{|c|c|c|}
\hline $\begin{array}{l}\text { Grade } \\
\text { level }\end{array}$ & \multicolumn{2}{|c|}{ Type of evidence (adapted from the modality of the Centre for Evidence Based Medicine, Oxford) } \\
\hline 1a: & \multicolumn{2}{|l|}{ Systematic review of level $1 \mathrm{~b}$ studies } \\
\hline $1 \mathrm{~b}$ & \multicolumn{2}{|c|}{$\begin{array}{l}\text { Prospective independent blind comparison (separate readers) of an appropriate spectrum/number of } \\
\text { consecutive patients, all of whom have undergone both the diagnostic test and the reference standard }\end{array}$} \\
\hline $2 a$ & \multicolumn{2}{|c|}{ Systematic review of level $2 \mathrm{~b}$ or level 4 studies } \\
\hline $2 b$ & \multicolumn{2}{|c|}{$\begin{array}{l}\text { Study performed in a set of non-consecutive patients or confined to a narrow spectrum/number of pa- } \\
\text { tients all of whom have undergone both the diagnostic test and the reference standard }\end{array}$} \\
\hline 3 & \multicolumn{2}{|c|}{ Systematic review of case-control studies (no intra-individual comparison) } \\
\hline 4 & \multicolumn{2}{|c|}{$\begin{array}{l}\text { Reference standard was not valid (unobjective, unblinded or not independent) or study was performed in } \\
\text { an inappropriate spectrum / number of patients }\end{array}$} \\
\hline 5 & \multicolumn{2}{|c|}{ Expert opinion } \\
\hline \multicolumn{3}{|c|}{ This scale will be also translated into a synthetic judgement of strength of recommendation using the following scale: } \\
\hline A & $\begin{array}{l}\text { Recommendation based on good evi- } \\
\text { dence }\end{array}$ & $\begin{array}{l}\text { At least one multicentre level } 1 \text { or } 2 \text { study } \\
\text { or } \\
\geq 3 \text { monocentric level } 1 \text { or } 2 \text { studies }\end{array}$ \\
\hline B & $\begin{array}{l}\text { Recommendation based on moder- } \\
\text { ate evidence }\end{array}$ & $\begin{array}{l}\text { One of the following: } \\
<3 \text { monocentric level } 1 \text { or } 2 \text { studies } \\
1 \text { level } 3 \text { study (systematic review) } \\
1 \text { multicentre level } 4 \text { study } \\
\geq 3 \text { monocentric level } 4 \text { studies }\end{array}$ \\
\hline C & $\begin{array}{l}\text { Recommendation based on experts } \\
\text { opinions }\end{array}$ & $\begin{array}{l}\text { Consensus of experts not supported by sufficient data of evi- } \\
\text { dence level A or B. }\end{array}$ \\
\hline 0 & No indication & \\
\hline $\mathrm{X}$ & Contraindication & \\
\hline
\end{tabular}

Table 1 Criteria for the assess ment of the level of evidence for the EFSUMB non-liver CEUS guidelines. Adapted from: Centre for Evidence Based Medicine, Oxford (http://www.essentialevidenceplus. com/product/ebm_loe.cfm?show= oxford).
C: The recommendation is based on expert opinion and panel consensus.

$\mathrm{X}$ : There is evidence that the intervention is harmful

0 : We added level 0 for situations in which not enough information was available to provide a recommendation.

In addition to the level of the recommendation (A, B, C, X or 0 ), we reported the strength of the recommendation based on the study with the highest quality supporting the recommendations, according to the classification detailed in $\bullet$ Table 1 .

\section{General Considerations}

$\nabla$

Any CEUS examination should be preceded by careful assessment of the target with conventional B-mode US and, when appropriate, with Doppler (spectral and/or colour/power). Before starting any CEUS examination, all relevant clinical information, including laboratory tests and previous imaging investigations, useful for reaching the expected final diagnosis, should be reviewed. This also helps to explain the expected benefit of the proposed CEUS study to the patient, an important step in obtaining consent.

Four transpulmonary UCAs are currently approved by the European Medicines Agency for use in European countries. The products and indications may vary on other continents.

- Levovist ${ }^{\circledR}$ (air with a galactose shell and palmitic acid as a surfactant) (Bayer-Shering Pharma AG, introduced in 1996). Production of Levovist has been discontinued and its use is not considered in these Recommendations.

- Luminity ${ }^{\circledR}$ (perflutren) (octafluoropropane with a phospholipid shell) (Lantheus Medical Imaging, inc., introduced in 2006). Sole European indication to date is cardiac which is outside the scope of these recommendations.

- Optison ${ }^{\circledR}$ (octafluoropropane - perflutren with an albumin shell) (GE Healthcare Inc., introduced in 1998). Sole European indication to date is cardiac which is outside the scope of these recommendations.
- SonoVue ${ }^{\circledR}$ (sulfur hexafluoride with a phospholipid shell) (Bracco Spa, introduced in 2001). Registered indications in Europe are cardiac, macrovascular, liver and breast lesions. This is the main agent in general use.

The composition, packaging, storage, indications and contraindications of these agents are detailed in the respective webpages of the manufacturers, whose addresses can be reached following the informations reported in Appendix 1.

Other UCAs are approved outside Europe and several more are under investigation.

Dose of contrast agent and data storage. For SonoVue ${ }^{\circledR}$, which is the focus of the current document and which is the UCA used in almost all of the referenced articles, the recommended dose is 2.4 $\mathrm{mL}$. This can be increased to $4.8 \mathrm{~mL}$ or decreased to $1.0 \mathrm{~mL}$ or less depending on the sensitivity of the equipment used, the type of transducer and the organ under investigation. When using higher frequency transducers, a dose of $4.8 \mathrm{~mL}$ performs better. A real-time videoclip should be recorded, preferably digitally, for review and documentation. The clip should include at best the whole examination or at least the most relevant parts, usually corresponding to the initial 10 - 40 seconds starting at the onset of enhancement with additional shorter clips taken at $2-3$ minutes. Finally, either the entire clip (preferred choice) or at least various selected most relevant frames should be stored permanently [6-9].

\section{Equipment}

Contrast-specific US modes are required and are generally based on the cancellation and/or separation of linear US signals from tissue and the utilisation of the non-linear response from microbubbles.

The non-linear response from microbubbles arises from two different mechanisms: 
- Non-linear response from microbubble oscillations at low acoustic pressure, chosen to minimize the disruption of the microbubbles.

- High-energy broadband non-linear response arising from microbubble disruption.

Non-linear harmonic US signals also arise in tissues themselves from distortion of the sound wave during its propagation through the tissue. The extent of this tissue harmonic response increases with the acoustic pressure, which is proportional to the MI. The precise unit of measurement for acoustic pressure is the Pascal, but the most common reference unit is the MI. Minimisation of bubble disruption is the main reason for using low MIs for real time imaging but it also reduces tissue harmonics and artefacts, thus facilitating the separation of signals from UCAs from those of tissue. Low MI is typically below 0.3 , but most equipments work well at MIs much below this (for details see manufacturers' recommendations or contact the manufacturers' representatives). For the purpose of this document, CEUS is defined as low MI real time contrast specific imaging, unless otherwise specified. This is in agreement with the terminology of the vast majority of the literature.

Technical information on US equipment capable of CEUS imaging can be accessed via the respective webpages reachable through the web address reported in Appendix 1. Updating this information is the responsibility of the companies.

EFSUMB is unable to express any judgement on the quality of particular scanners or transducers. However, the following features should be kept in mind, as they characterise the quality of the equipment used for CEUS examinations:

Sensitivity reflects the ability of a system to detect extremely small amounts of microbubbles. Good sensitivity extends the duration of useful enhancement.

When using high frequency probes, higher doses of UCA may be needed to achieve a sufficient enhancement level, as these frequencies are higher than those at which current UCAs resonate most strongly.

The ability to image small differences in local contrast concentration is a component of sensitivity. It reflects the dynamic range of contrast performance.

Tissue suppression is mandatory to differentiate contrast enhancement from tissue echoes. Strongly reflective structures such as vessel walls, the abdominal wall and gas-filled structures can break through and appear on the CEUS part of the screen.

Resolution. As in B-mode, temporal and spatial resolution are important for CEUS imaging. Temporal resolution is defined by the frame rate at a given line density, depth and width. A high frame rate allows visualisation of the flow direction in arteries. It may also produce more rapid destruction of bubbles within the acoustic field. Spatial resolution mainly refers to the ability to display bubble echoes with optimal detail. Additionally, the image should be homogeneous throughout.

Like all imaging techniques, CEUS has artefacts, mainly caused by incorrect machine settings or UCA dosages [10]. The MI is the most important setting for CEUS and gain is the second one. Adequate training allows knowledge, recognition and prevention of artefacts.

\section{Investigator Training}

$\nabla$

Competence ensured by adequate training is a prerequisite to achieve correct diagnoses when using ultrasonography and especially CEUS. EFSUMB has defined three levels of training in its minimal training requirements [11] (EFSUMB Appendix 14) [12] and recommends that CEUS should be performed by operators at a competence level higher than Level 1 . To ensure adequate training, EFSUMB started a new initiative to organise dedicated Euroson Schools on CEUS in 2009 [13].

EFSUMB advises that investigators intending to start using CEUS spend some time under the supervision of an expert, who is preferably at Level 3. It is important that there is a sufficient number and variety of examinations in their own department. Investigators should also consult the manufacturers to ensure that their scanner is optimised for CEUS examinations. The practice of CEUS also requires knowledge of UCA administration and contraindications and the skill to handle possible side effects within the medico-legal framework of their country.

\section{Terminology}

$\nabla$

UCAs were initially developed to enhance Doppler US and any scanner with Doppler facilities can be utilised for this. Conventional Doppler US operates at a higher acoustic power than realtime contrast-specific modes. Consequently microbubble disruption occurs at a high rate and the enhancement does not last long (a few minutes) after bolus injection. Moreover, the injection of contrast as a bolus often produces an initial "blooming" effect (proportional to the amount of contrast utilised), so that either smaller amounts or a continuous infusion ( $1-2 \mathrm{ml}$ per minute) may be preferable. This technique has few applications nowadays since CEUS can provide information either more accurately or in an easier way in most organs.

During CEUS, the screen should show only a few signals from intensely reflective structures, such as signals generated by the large difference in acoustic impedance between the lung and the diaphragm (overload of the tissue suppression software). This applies both to single-screen contrast-only displays and to the split-screen display, where the CEUS image is shown alongside a low MI version of conventional B-mode image. In some equipment a single panel mixed mode is used. In these cases, the underlying conventional B-mode image is seen, with the CEUS overlay displayed in a different colour, potentially with the addition of colour Doppler information.

The description of the behaviour of tissue or lesion under examination should be in terms of its enhancement, taking into consideration its temporal behaviour, degree of enhancement, and contrast distribution.

1. As regards the timing of enhancement, two phases are apparent for most organs:

a) the arterial phase, starting from the first arrival of contrast (usually in $10-20$ seconds) until around $30-45$ seconds, during which the degree of enhancement increases progressively. b) the venous phase which starts from approximately $30-45$ seconds after contrast injection, during which the degree of enhancement shows a plateau and then decreases progressively until microbubble signals have disappeared completely or fallen to the noise level. Most organs have a single blood supply with a single inflow (namely arterial) phase, the exceptions being the liver, which is supplied by its artery and the portal vein, leading to two distinct inflow phases (arterial and portal venous) and the lungs, which are supplied by pulmonary and bronchial arteries with different arrival times. 
The liver and spleen are also exceptions in that they tend to retain microbubbles longer than other organs, probably due to the of trapping of microbubbles in their unique microcirculations after clearance from the remainder of the macrovasculature. Consequently, the wash-out phase in most organs is shorter than in the liver and spleen, whose prolonged retention is termed the late phase. There is no precisely definable event that allows an exact distinction between the arterial and venous and late phases.

The time of contrast arrival is usually 10 to 20 seconds after i. v. injection, but factors such as a slow injection of microbubbles in very peripheral small veins or cardiac diseases may prolong it, whereas intracardiac or pulmonary shunting or a hyperdynamic circulation may shorten it.

2. The degree of enhancement is difficult to assess, unless measurements are made using in-built or off-line software, for example, in the assessment of tumour response to antiangiogenesis treatment [14]. Generally, when the target of the study is a focal region in a parenchymal organ, the degree of enhancement should be compared to the surrounding parenchyma or to the paired organ when available. The lesion might be relatively hyperenhancing, isoenhancing, hypoenhancing or nonenhancing and the pattern should be described separately for the arterial and venous phases. The transition from hyper- or iso-enhancement to hypoenhancement is commonly referred to as "wash-out". The use of this term for other enhancement patterns creates confusion and should be avoided. EFSUMB recommends that reports on CEUS are made in terms of the degree of enhancement. For targets that have no background tissue to describe the relative enhancement (studies of large vessels, endocavitary administration, or when a mass occupies an entire organ, etc), it is important to describe the presence or absence of enhancement and its distribution (e.g. in septa within a complex cystic mass or a fluid-containing structure, or blood flow where this is not expected to be present, for instance outside an organ) whereas the degree of enhancement is more difficult to assess.

3. Contrast distribution. When enhancement is seen, its distribution should be described. The relevant terms have not been predetermined and should be chosen with the aim of characterising the tissue and reaching a diagnosis. The description of whether the enhancement is homogeneous or heterogeneous and, in the latter case, if non-perfused regions exist, should be included. Any further description of the enhancement pattern is left to the operator inspired by the recommendations for each organ. In general terms, the CEUS depiction of non-perfused (potentially necrotic or liquid) areas might be relevant prior to any US-guided biopsy in order to better identify the target.

\section{Safety}

In general, UCAs are very safe with a low incidence of side effects. They are not nephrotoxic and do not interact with the thyroid gland and it is therefore not necessary to perform laboratory tests before their administration. The incidence of severe hypersensitivity or anaphylactoid reactions is lower than with current X-ray agents and is comparable to that of MR contrast agents. UCAs are not licensed in pregnancy. Breastfeeding is a contraindication in some countries.
Life threatening anaphylactoid reactions have been reported with a rate of less than $0.002 \%[15,16]$. Previous allergic/anayphylactoid reaction to X-ray iodinated contrast agents does not necessitate the prophylactic use of steroids or antihistamines prior to UCA injection since the two types of agent are completely different.

There is a theoretical possibility that the interaction of diagnostic US and UCA could produce bioeffects. In vitro cellular effects that have been observed include sonoporation, haemolysis and cell death. Although observed in vitro, such bioeffects may have relevance for the in vivo situation as they result from interactions between gas bodies and cells. Data from small animal models suggest that glomerular capillary haemorrhage and other microvascular rupture could occur when microbubbles are insonated at high MI[16]. This could be injurious in specific situations in which such vascular damage would be clinically important such as in the eye and brain. This potential for non-thermal bioeffects exists with all modes, including conventional 2D imaging and 3D methods. The MI provides a useful, albeit very rough, on-screen indicator of the potential for non-thermal effects. Changes have been observed in vivo in mammalian tissue models for diagnostic US exposures with an MI greater than $\sim 0.4$ in the presence of UCAs. In addition, premature ventricular contractions have been described when high MI end systolic triggering is used in echocardiography $[17,18]$.

Users should balance the potential clinical benefit of the use of UCAs against the theoretical possibility of associated adverse bioeffects in humans.

Some general recommendations are:

- Resuscitation facilities should be available.

- Caution should be exercised for off-label use of UCA in tissues in which damage to the microvasculature may have serious clinical implications, such as in the eye, the brain and in the neonate.

- As in all diagnostic US procedures, the operator should be mindful of the desirability of keeping the displayed MI low and of avoiding unduly long exposure times. Caution should be exercised when using UCAs in patients with severe coronary artery disease and pulmonary hypertension. Unstable ischaemic heart disease in the 7 days prior to administration is a relative contraindication. Caution with respect to the use of UCAs in these cardiac instances derives from an anecdotal temporal, but unproven causal, association between contrast injection and death in severely compromised cardiac patients. However, in very large patient cohorts, the use of UCAs for acute cardiac patients has been shown to be associated with a decreased, not increased risk of death, thanks to the efficacy of the modality $[19,20]$.

\section{Paediatrics}

$\nabla$

The current use of CEUS in paediatric work-up in Europe is peculiar. Two UCAs are registered for clinical non-cardiac use, Levovist $^{\circledR}$ and SonoVue ${ }^{\circledR}$. However only Levovist ${ }^{\circledR}$ is approved for use in children and adolescents and only for the indication of vesico-ureteral reflux study. At the same time production of Levovist $^{\circledR}$ has ceased and it is no longer available. SonoVue ${ }^{\circledR}$, performs equally well for this particular indication, but has to be used off-label, as previously mentioned. 
CEUS in paediatric applications remains of critical importance, because of its obvious benefits compared to alternative imaging modalities, which in most cases necessitate exposure to ionizing radiation and the use of potentially harmful contrast agents. The benefit of avoiding ionizing radiation is clearly far more important in children and adolescents than in adult patients. This important question was addressed recently in a Letter to the Editor of Ultraschall in der Medizin/European Journal of Ultrasound, with a reply from the EFSUMB Executive Board [5]. There is no easy solution to this problem as strict rules and regulations apply to the official registration of medical drugs, which is of course for the safety of patients and thus to the benefit of all, including the medical community and pharmaceutical producers. In some situations, however, strict regulations may inhibit use of the most beneficial and simple techniques or drugs, and CEUS is one such example.

\section{Pancreas}

$\nabla$

\subsection{Background}

CEUS is not indicated for the detection of focal solid or liquid pancreatic lesions, but can be utilised for the characterisation of sonographically detected lesions [21-23]. The use of CEUS improves the diagnostic accuracy of US in the study of pancreatic pathologies.

\subsection{Study Procedure}

CEUS is an accurate imaging method, superior to Doppler techniques, for the visualisation of intrapancreatic vessels and microvessels [24]. Enhancement of the pancreas begins immediately after aortic enhancement. After this early arterial phase (10 to $30 \mathrm{sec}$ ), there is a transient venous phase (30 to approximately $120 \mathrm{sec}$ ), as with other dynamic imaging modalities [4].

The CEUS study of a pancreatic mass should aim at its characterisation and the evaluation of its relationship with the peripancreatic arteries and veins $[21,22,25,26]$. After completion of the pancreatic study, the liver should be assessed in the late phase, exploiting the same contrast injection, searching for metastases [22].

\subsection{Pancreatic Masses}

\subsubsection{Image interpretation for lesion characterisation}

The enhancement pattern of focal pancreatic lesions is usually compared with the adjacent pancreatic tissue. Therefore, the field of view should include the mass under investigation together with a portion of surrounding pancreatic parenchyma.

\subsubsection{Adenocarcinoma}

Ductal adenocarcinoma, the most common primary malignancy of the pancreas, is typically hypoenhancing in all phases, presumably because of the desmoplastic reaction and low mean vascular density [27-29]. This pattern is reported in about $90 \%$ of cases $[23,27,30]$. Lesion size and margins as well as the relationship with peripancreatic vessels are better visualised with CEUS than with conventional US [25, 26]. CEUS can demonstrate changes in pancreatic tumour vascularisation during chemotherapy [31,32] raising a hope for future use in clinical practice.

\subsubsection{Neuroendocrine tumours}

Neuroendocrine tumours typically present as hyperenhanced masses in the arterial phase, owing to their abundant arterial vascularisation, even when the Doppler study is negative [21, $33,34]$. In larger tumours necrotic avascular areas result in inhomogeneous enhancement $[33,34]$.

\subsubsection{Mucin-producing cystic tumours}

CEUS improves the ultrasonographic differential diagnosis between pseudocysts and cystic tumours of the pancreas by accurately revealing vascularisation of intralesional septa or nodules [22].

Mucinous cystadenoma is a potentially malignant lesion that may transform into cystadenocarcinoma. Usually unilocular, it appears as a round macrocystic lesion, with particulate content, irregular thick walls and internal inclusions which enhance on CEUS $[30,35]$. In general, mucinous cystic pancreatic tumours have vascularised septa and parietal nodules [22, 35]. Intraductal papillary mucinous neoplasms (IPMN) are divided into main-duct and side branch-duct types. CEUS is helpful for differentiating between perfused (nodules) and non-perfused (clot) regions [36]. Magnetic resonance imaging (MRI) and endoscopic US (EUS) are the imaging methods of choice for the study of this tumour to demonstrate the communication of the cystic lesion with the pancreatic ducts [37, 38]. CEUS can be employed in the follow-up of borderline cystic lesions of the pancreas, if well visualised on US, in order to reduce the use of MRI.

\subsubsection{Serous cystadenoma}

Serous cystadenoma is a benign cystic lesion, typically with a lobulated microcystic appearance with thin and centrally oriented septa, which are vascularized on CEUS [22]. When the cysts are minute, microcystic serous cystadenoma may mimic a solid lesion, both on conventional US and CEUS. They are hyperenhanced on CEUS [38].

\subsubsection{Pseudocysts}

Pseudocysts typically contain non-vascularised material (debris), with the exception of transversing vessels, which are typically found in the early stages. Pseudocysts do not show any signal on CEUS and remain completely non-enhancing in all phases, even when they are heterogeneous on US [30, 35]. The reported sensitivity and specificity of CEUS in characterising pseudocysts is up to $100 \%$ [35].

\subsection{Pancreatitis}

In severe acute pancreatitis, CEUS may help identify and delineate necrotic areas, which do not enhance [39]. If the pancreatic region is clearly visible on US, CEUS can be used in the follow-up of acute pancreatitis after CT staging, in order to reduce the number of CT examinations [39].

Focal mass-forming pancreatitis and autoimmune pancreatitis have been reported to have similar enhancement to that of the normal pancreatic parenchyma [27].

\subsection{Recommended uses and indications}

Focal pancreatic lesions identified with US can be studied with CEUS in order to improve:

1. Characterisation of ductal adenocarcinoma. (Recommendation Level: A;1b) 
2. Differential diagnosis between pseudocysts and cystic tumours. (Recommendation Level: A;1b)

3. Differentiation of vascular (solid) from avascular (liquid/necrotic) components of a lesion. (Recommendation Level: A;1b)

4. Defining the dimensions and margins of a lesion, including its relationship with adjacent vessels. (Recommendation Level: $\mathrm{B} ; 2 \mathrm{~b})$

5. Management of the lesion with a better distinction between solid and cystic lesions, thus providing information for the choice of the next imaging modality (i.e. MRI and/or Endoscopic US for cystic lesions). (Recommendation Level: C;5)

6. Diagnosis in cases that are indeterminate on CT (vascularisation of solid pancreatic lesions; differential diagnosis between pseudocysts and pancreatic cystic tumours, especially mucinous cystic tumour). (Recommendation Level: C;5).

\section{Contrast Enhanced Endoscopic US (CE-EUS)}

\subsection{Background}

Contrast enhanced endoscopic ultrasound (CE-EUS) is a newly established method which combines the advantage of high resolution US of internal organs with the administration of UCAs [38]. CE endoscopic Doppler US works at high MI that does not require specific software and uses the UCA as a Doppler enhancing medium [40, 41]. Low MI CE-EUS has been introduced recently using the same contrast-specific modes as for transabdominal CEUS [42-44].

EUS will be performed first and CE-EUS can be added to characterise internal vasculature, to differentiate benign from malignant masses, and to improve staging and real-time guidance of diagnostic and therapeutic procedures.

\subsection{Study procedures}

\subsubsection{Contrast enhanced high mechanical index EUS}

In CE endoscopic Doppler US the colour Doppler window should include the whole tumour if possible. The Doppler frequency should be as high as possible, depending on the distance to the tumour. The Doppler scale should be between 3 and $8 \mathrm{~cm} / \mathrm{s}$ assuming an insonation angle of $0^{\circ}$ if directional colour Doppler is used [45]. The gain should be as high as possible without inducing artefacts. The flow velocity range (pulse repetition frequency, PRF) should be $5-15 \mathrm{~cm} / \mathrm{s}$ if pulsed wave Doppler is used. The technique is the same as for transcutaneous US. CE endoscopic Doppler US scanning takes an extra 3-4 min [46].

\subsubsection{Contrast enhanced low mechanical index EUS (low MI CE-EUS)}

The technique is the same as described for the transcutaneous route. However, there are various settings that should be checked in accordance with the manufacturer. The MI used in published feasibility articles was variable [42, 43, 47], ranging from 0.08 to 0.3 .

\subsection{Image interpretation for pancreatic lesions}

\subsubsection{Discrimination of adenocarcinoma}

CE endoscopic Doppler US can be used to differentiate between pancreatic neoplasms, mainly the hypoenhancing ductal adenocarcinoma and the iso- or hyperenhancing neuroendocrine tumours but also pseudo-solid lesions like serous microcystic cystadenoma [21, 37, 48]. The differential diagnosis between ductal adenocarcinoma and mass-forming focal pancreatitis can be improved since adenocarcinomas mainly contain arterioles and hardly any venules whereas in focal pancreatitis both arterioles and venules are detectable during CE endoscopic Doppler US [45, 49-51]. The method can be improved further by estimation of the resistance index (RI) of the arterioles. The cut-off RI for suspected malignant lesions is $>0.70$ versus $<0.70$ for non-malignant, inflammatory lesions [52]. As for all other US applications, the limitations of CE endoscopic Doppler US are extensive calcifications and poor B-mode depiction of the lesion.

Low MI CE-EUS seems to be more powerful, but there is currently only minimal published data [53-55]. Analysis of the microvessel architecture seems to be an advantage over transcutaneous CEUS, e.g., the central feeding artery in serous cystadenoma [21]. The technique can be used in cases in which significant artefacts impede transcutaneous visualisation, especially for characterisation and follow-up of focal pancreatic lesions. The accuracy for the differential diagnosis of pancreatic adenocarcinoma is reported to be over $85 \%[53,55,56]$.

\subsubsection{Discrimination of pancreatic pseudocysts from cystic tumours}

Low MI CE-EUS can be also used for differential diagnosis in cystic pancreatic lesions [57] as described in the transcutaneous section. EUS has a clear advantage in small cystic lesions and in patients with impaired transcutaneous visibility.

\subsection{Differential diagnosis of autoimmune pancreatitis} Both focal and diffuse autoimmune pancreatitis show hyperenhancement. Low MI CE-EUS is able to visualise hyperenhancement to discriminate ductal adenocarcinoma (which does not hyperenhance) from autoimmune pancreatitis [58] but experience is limited. 3D reconstruction might have a higher impact because of better visualisation of vessel patterns [58].

\section{Recommended uses and indications}

CE-EUS can be recommended for:

1. Discrimination of hypoenhancing ductal adenocarcinoma of the pancreas from other iso- or hyperenhancing lesions. Experience is mainly based on CE endoscopic Doppler US. (Recommendation Level: A;1b)

2. Discrimination of mass-forming chronic pancreatitis from ductal adenocarcinoma in patients with chronic pancreatitis. (Recommendation Level: A;1a)

3. Improved discrimination of cystic tumours from pancreatic pseudocysts. (Recommendation Level: A;1b)

\section{Gastrointestinal Tract}

$\nabla$

\subsection{Background}

When examining the intestines, it is preferable to use frequencies above $7.5 \mathrm{MHz}$ to enable optimal visualisation of wall layers, thickened bowel walls and lesions. Higher contrast doses (e.g. $2.4-4.8 \mathrm{ml} \mathrm{SonoVue}{ }^{\circledR}$ ) should be used. The arrival time in the intestinal capillaries is usually $10-20 \mathrm{sec}$ after injection, particularly in the submucosal layer [59]. Peak enhancement is reached after $30-40 \mathrm{sec}$ and this arterial phase is followed by a venous phase that lasts from 30 to approximately $120 \mathrm{sec}$. 


\subsection{Study procedure}

Before i.v. UCA administration, the intestines must be examined in B-mode and Doppler US to detect any pathology. The difference in bowel wall microvascularity between healthy and diseased intestines can be recognised by CEUS [60].

\subsection{Inflammatory Bowel Disease (IBD)}

\subsubsection{Image interpretation and evaluation}

CEUS allows the quantification of bowel wall vascularity in patients with Crohn's disease [61] and correlates well with MRI of the intestinal wall [62, 63]. Additionally, CEUS visualises the microvascularity of the tissue surrounding the intestines. Time Intensity Curve (TIC) analysis may further help to quantify disease activity.

\subsubsection{Disease activity}

US can be used to study disease activity in IBD using bowel wall thickness and Doppler measurements as a marker of inflammation [64-66]. Adding a UCA improves reliability in estimating disease activity in Crohn's disease [67, 68]. Enhancement in different wall layers can be evaluated and quantified in Crohn's disease and correlates to clinical activity indices with good sensitivity and specificity [69-71]. Quantitative measurements of bowel enhancement obtained by CEUS also correlate with endoscopic severity [72] and histopathologic findings [71]. Furthermore, ultrasonographic evaluation of the changes of bowel wall enhancement during biological therapy (e.g. antiTNF agents) promises to be a useful and relatively cheap imaging modality for the clinical monitoring of the activity of Crohn's disease [73].

\subsubsection{Distinguishing between fibrous and inflammatory strictures}

Preliminary studies indicate that the use of UCAs is effective for the recognition of predominantly cicatricial stenoses in patients with Crohn's disease [74]. In patients with bowel stricture and resultant obstruction, it is important to determine whether there is active inflammation at the site of stricture or if the stenotic segment is fibrotic. Using CEUS, the active inflammatory components enhance strongly whereas a fibrotic stricture enhances poorly [75].

\subsubsection{Abscesses}

Distinguishing abscesses from inflammatory infiltrates is an important clinical task in the management of Crohn's disease. If areas of a significant size close to or within an affected bowel wall are completely devoid of microbubble signals, these lesions likely represent abscesses rather than inflammatory infiltrates, particularly if adjacent tissue shows hyperenhancement [61]. Similar findings also apply to abscesses caused by other diseases, e.g. diverticulitis [76].

\subsubsection{Fistula}

Injecting a UCA into one of the orifices of a fistula improves visualisation of the course of the fistula in Crohn's disease [77]. The UCA can be diluted manyfold with sterile saline before injection into orifices (see section on intracavitary applications).

\subsection{Limitations}

It is impossible to visualise all bowel segments using transabdominal US. Intestinal peristalsis can impair image quality and the repeatability of quantitative measurement of bowel enhancement patterns. More studies are needed to establish the exact role of CEUS in the imaging of GI pathology [78].

\section{Recommended uses and indications}

CEUS is indicated in the following clinical situations:

1. Estimation of disease activity in inflammatory bowel disease. (Recommendation Level: $\mathrm{B} ; 1 \mathrm{~b}$ )

2. Discerning between fibrous and inflammatory strictures in Crohn's disease. (Recommendation Level: C;4)

3. Characterisation of suspected abscesses. (Recommendation Level: $\mathrm{C} ; 4)$

4. Confirming and following the route of fistulas. (Recommendation Level: C;4)

\section{Spleen}

\subsection{Background}

The indication for CEUS investigation of the spleen, other than in trauma (see section 15), is the characterisation of focal lesions [79]. Solid splenic lesions may be occasional incidental US findings in asymptomatic subjects. In this setting and when they are single or scanty, especially if they are hyperechoic on conventional US, they are benign in the large majority of cases. However, in cancer patients, especially when lesions are multiple, hypoechoic and of recent onset, they tend to be malignant. Unfortunately, the clinical information and US findings are not usually sufficient to provide a reliable distinction between benign and malignant masses. Fine needle biopsy of splenic masses may have complications, especially peritoneal bleeding, making a noninvasive diagnosis highly desirable. CEUS may increase confidence in suggesting a benign nature.

\subsection{Study procedure}

The peculiar splenic vascularisation [80] leads to a rapid and transiently inhomogeneous enhancement pattern, especially in congestive splenomegaly, resembling the "zebra" pattern seen on dynamic CECT or CEMRI. About 1 minute after injection, the parenchyma becomes homogeneous, showing strong and persistent late phase enhancement that lasts longer than 5 minutes [81].

\subsection{Image interpretation}

CEUS is useful for characterising ectopic splenic tissue (accessory spleens and post-splenectomy splenosis) [82]. Splenic hilar lymph nodes, adrenal lesions, pancreatic tail tumours, metastatic deposits, and other lesions show less enhancement than the normal splenic parenchyma in the late phase. Accessory spleens usually exhibit a typical location and appearance and further imaging is necessary only in a minority of cases. CEUS shows an enhancement pattern exactly paralleling that of the adjacent spleen [83], i.e. more intense than that of other organs in the late phase.

In splenic infarction, CEUS accurately demonstrates the shape and extent of the lesion as a wedge-shaped, non-enhancing area based on the spleen capsule and pointing towards the hilum [84].

A splenic abscess is a poorly- or completely non-enhancing lesion, best recognised in venous phase images, with an enhancing rim and septa. No contrast enhancement is seen within 
the internal fluid, debris, or necrotic components. Intermediate and early stage lesions show mixed patterns, like those in the liver [85].

Typical findings for benign splenic lesions include either no contrast enhancement or rapid wash-in, followed by persistent enhancement in the late phase. If CEUS findings are consistent with a benign splenic lesion, CT seems to be of limited additional value [86]. In contrast, the combination of contrast enhancement (diffuse or peripheral) in the arterial phase followed by rapid and marked wash-out is typical for malignant lesions (metastases or lymphoma) [87-90]. However, the behaviour of malignant and some benign lesions partially overlap, since haemangiomas, hamartomas, as well as other uncommon splenic abnormalities, may show some degree of wash-out, mimicking a malignant pattern $[90,91]$.

\subsection{Differential diagnosis and limitations}

In patients with left upper quadrant pain and splenic inhomogeneity on conventional US, CEUS allows visualisation of the underlying splenic abnormalities [84] increasing operator confidence in establishing a diagnosis [92]. CEUS is particularly helpful when an infarcted region is barely recognisable on US or it is spherical and simulates a focal lesion. Additionally, CEUS can diagnose splenic tumour infiltration mimicking infarction on conventional US. Differentiating splenic abscesses from necrotic lymphomatous lesions remains a challenge, since both conditions may occur in the immunocompromised patient.

CEUS may be helpful when a splenic cyst with ill-defined margins and/or inhomogeneous content is identified with US. Echogenic splenic tumours incidentally found on US and assessed by CEUS can be diagnosed as haemangiomas and can be managed with follow-up examinations.

\subsection{Splenic tumour detection}

CEUS may aid in detecting focal lesions in the spleen [79]. Lesions that are not seen on baseline US can be revealed by CEUS, which has been found to be $90 \%$ sensitive and $100 \%$ specific compared to CT with respect to lesion detection in lymphoma patients [93]. In a retrospective study on splenic metastases, CEUS increased the detection rate by $38 \%$ [94]. Lesions found in patients with solid organ or haematological malignancies require whole-body imaging using CT and/or PET [95], but CEUS can be useful if they are inconclusive, particularly to rule out lesions when PET shows "non-specific uptake". Finally, CEUS can be used in patients with splenic malignancies to monitor their response to treatment. When chemotherapy is successful, the lesion becomes almost nonenhancing on CEUS with absence of any intra-nodular vessels or microcirculation. These findings are seen early, paralleling the PET response. Long-term follow-up shows progressive disappearance of the lesions.

\section{Recommended uses and indications}

CEUS can be utilised:

1. To characterise splenic parenchymal inhomogeneity or suspected lesions on conventional US. (Recommendation Level: $\mathrm{B} ; 1 \mathrm{~b})$

2. To confirm suspected splenic infarction. (Recommendation Level: $\mathrm{B} ; 2 \mathrm{~b}$ )

3. To characterize suspected accessory spleens or splenosis. (Recommendation Level: B;2b)
4. To detect splenic malignant lesions in oncologic patients when CT and/or MRI and PET are contraindicated or inconclusive. (Recommendation Level: B;1b)

\section{Kidney}

\subsection{Background and vascular anatomy}

In most centres, ultrasonography is the preferred first imaging modality in patients with known or suspected renal disease. The main objectives are to measure renal size, to detect focal lesions and obstruction of the collecting system and to look for vascular disorders. However, US is not reliable in distinguishing between different types of tissue and benign and malignant lesions may be difficult to distinguish. Doppler helps to characterise renal blood flow, with limitations because of attenuation, poor sensitivity for very slow blood flow, and angle dependency.

\subsection{Study procedure}

Kidneys enhance quickly and intensively after microbubble administration. The arterial pedicle and main branches enhance first, followed within a few seconds by complete fill-in of the cortex. Unlike with Doppler US, signals from microbubbles are independent of the angle of insonation, and depiction of renal perfusion is excellent even at the renal poles. Medullary perfusion follows cortical perfusion, with the outer medulla enhancing first, followed by gradual fill-in of the pyramids [96]. There is no microbubble excretion into the urinary tract. As contrast concentration in the general circulation decreases, enhancement fades. The expert opinion is that contrast enhancement is less intense and fades earlier in patients with chronic renal disease.

\subsection{Renal ischaemia}

Several investigations, both in animal studies and in humans, have shown excellent diagnostic performance in the detection of renal parenchymal ischaemia, similar to that of CECT [97] and superior to colour Doppler US, making CEUS a recommended technique in case of suspicion of infarction. Infarcts appear as wedge-shaped non-enhancing areas within an otherwise enhanced kidney. The excellent spatial resolution of CEUS allows confident differentiation between renal infarction and cortical necrosis, which appears as non-enhancing cortical areas with preserved hilar vascularity $[96,98]$.

Another important application of CEUS in patients with renal ischaemia is differentiation between non-perfused, infarcted tissue and hypoperfused parenchymal regions. On Doppler US, both appear as areas lacking colour signal, but only infarcted areas completely lack contrast enhancement after microbubble injection.

\subsection{Renal artery stenosis}

UCAs can be injected to improve the sensitivity of conventional colour Doppler US in identifying the renal arteries, with an improvement of approximately $10 \%$ [99]. This allows correct positioning of the sample volume for the detection of Doppler spectral tracings with the aim of screening for renal artery stenosis. Routine use of UCA offers no significant advantage [99]. 


\subsection{Renal focal lesions}

12.5.1 Differential diagnosis between cystic and solid lesions Thanks to its excellent sensitivity, CEUS is more sensitive than CECT in detecting blood flow in hypovascularized lesions [100] and can be utilised in the few cases in which the distinction between solid hypovascular tumours (showing enhancement, even if there is very little flow) and atypical cystic masses (no contrast perfusion at all) remains unresolved after CT and colour Doppler US.

\subsubsection{Differential diagnosis between solid renal masses and pseudotumours}

The enhancement in renal tumours differs from the surrounding parenchyma, with a difference in degree or distribution of enhancement in at least one vascular phase, in the majority of cases. This helps differentiation between tumours and pseudotumours, since the latter have the same enhancing characteristics as the surrounding parenchyma in all phases $[96,101]$. Solid renal tumours do not show diagnostic perfusion patterns on CEUS, which is thus usually not able to differentiate between malignant and benign solid renal masses (e.g. carcinoma versus angiomyolipoma).

In expert hands, CEUS may help identify renal vein invasion by cancers with an accuracy comparable to CECT [102]. In addition, in the opinion of the experts, the arterial vascularisation of the thrombus in the renal vein promises to differentiate bland thrombus (non-enhancing) from tumour invasion (enhancing thrombus), but further studies are necessary.

\subsubsection{Characterisation of complex cystic renal masses}

CEUS allows the characterisation of renal cystic lesions as benign or malignant with at least the same accuracy as CECT. It is more sensitive than $\mathrm{CT}$ in detecting enhancement of the cystic wall, septa, and solid components [103-106]. Nonetheless, CT remains the reference method for staging patients with malignant cystic lesions. CEUS is very well suited for the follow-up of non-surgical lesions and might replace CT, in light of its lack of contraindications, wide availability and lack of ionizing radiation, all of which are particularly welcome in this setting.

\subsubsection{Renal infections}

Following the European Urology Association guidelines, diagnosis of acute uncomplicated pyelonephritis is based on the clinical picture and laboratory findings. Conventional B-mode US is used to rule out urinary obstruction and renal stones. Additional investigations should be considered if the patient remains febrile after $72 \mathrm{~h}$ of treatment. These recommendations have a low level of evidence, and are made despite the absence of directly applicable clinical studies of good quality. As a consequence, the role of CEUS and of imaging itself in patients with uncomplicated pyelonephritis is debated and no clear indications exist, despite the finding that CEUS depicts renal abscesses as non-enhancing regions and can be used to monitor their course [96].

\subsection{Assessment of percutaneous ablation therapies}

Percutaneous ablation plays an increasing role in the management of patients with renal tumours. Diagnostic imaging in these patients includes CECT and/or CEMRI imaging during pre-treatment work-up and at distinct time points during follow-up after treatment. Unenhanced US can be used to guide ablation, but does not provide enough information about the outcome of ablation treatments. There is increasing evidence that, CEUS overcomes many of these limitations in patients undergoing renal tumour ablation [107]. It provides important information on lesion vascularity, and may improve visualisation of lesions that are poorly demonstrated on the baseline US scan. Guidance of the ablation needle into the lesion and assessment of the therapeutic results are improved [108, 109]. Similarly to the liver, the intra-procedural assessment must be delayed by $5-10$ minutes after the end of the ablation to allow the heat-generated gas and the related artefacts to dissipate. Any contrast enhancement within the ablation zone is conventionally regarded as residual tumour. Larger vessels surrounding the ablated region may be misinterpreted as residual tumour. Meticulous comparison with pre-procedural examinations and evaluation of lesion morphology are therefore important to avoid misdiagnosis, since residual tumour presents as nodular or crescent-like enhancing regions, with enhancement remaining similar to what observed in the tumour before treatment [110]. In two recent series of 28 [111] and 66 [110] renal tumours with sufficiently long follow-up, CEUS provided similar overall accuracy to that of CT/ MRI in confirming the adequacy of treatment.

\section{Recommended uses and indications}

CEUS is recommended in the following clinical situations:

1. Suspected vascular disorders, including renal infarction and cortical necrosis. (Recommendation Level: A;1a)

2. Differential diagnosis between solid lesions and cysts presenting with equivocal appearance at conventional US. (Recommendation Level: $\mathrm{B} ; 2 \mathrm{~b}$ )

3. Differentiation between renal tumours and anatomical variations mimicking a renal tumour ("pseudo-tumours") when conventional US is equivocal. (Recommendation Level: B;1b). However, both CEUS and CECT have limitations in rare very small isoenhancing tumours.

4. Characterisation of complex cystic masses as benign, indeterminate or malignant to provide information for the surgical strategy. (Recommendation Level: A;1b)

5. Additional aid, when necessary, in the follow-up of non-surgical complex masses. (Recommendation Level: C;5).

6. Identification of clinically-suspected renal abscesses in patients with complicated urinary tract infection. (Recommendation Level: C;5)

7. In patients undergoing renal tumour ablation under US guidance, CEUS may be used to improve lesion visualisation in difficult cases and to detect residual tumour either immediately or later after ablation. When CEUS is planned, pre-ablation assessment of lesion vascularity is important. (Recommendation Level: $\mathrm{B} ; \mathrm{1b}$ )

\section{Vesico-Ureteric Reflux (VUR)}

$\nabla$

\subsection{Background}

In addition to intravascular use, UCAs are suitable for intracavitary administration. The intravesical administration of UCA for the diagnosis of vesico-ureteric reflux, voiding urosonography, has become a routine option that can completely replace reflux examinations using ionizing radiation (i.e. voiding cystourethrography) in girls. Comparative studies between voiding urosonography and cystourethrography have revealed sig- 
nificantly higher sensitivity for urosonography in detecting reflux [112, 113].

Levovist $^{\circledR}$ is licensed for VUR. SonoVue ${ }^{\circledR}$, seems to perform at least as well [114] although it is not licensed for this indication.

\subsection{Study procedure}

The basic steps of voiding urosonography [112] are:

(a) US evaluation of the kidneys and bladder;

(b) intravesical administration of UCA diluted in normal sterile saline and

(c) repeated scanning of the bladder and kidneys at low MI during and after bladder filling and, finally while voiding. During the last step, urethrosonography (transpubic and/or transperineal) is added in some centres.

UCAs can be administered via a transurethral bladder catheter or via suprapubic puncture $\left(0.1-0.5 \mathrm{~mL}\right.$ SonoVue ${ }^{\circledR}$ in $500 \mathrm{~mL}$ $0.9 \%$ saline $[112,115])$, by slow instillation during CEUS monitoring, until strong enhancement of the bladder content has been achieved. The dose can be adjusted if there is excessive shadowing or insufficient signal. A full bladder is necessary for suprapubic puncture, and it is advisable to apply an anaesthetic plaster to the puncture site about one hour prior to the examination.

\subsection{Diagnosis of vesico-ureteral reflux}

Reflux is diagnosed when microbubbles appear in one or both ureters and/or the pelvicalyceal system. The severity of reflux is graded using 5 degrees (grade I-V) [116] comparable to the international reflux grading system of voiding cystourethrography. The US scan is continued during and after voiding with the child supine, prone, sitting, or standing, always scanning the kidneys alternately and also the bladder, if the patient's position allows [112].

\subsection{Voiding urosonography}

Voiding urosonography is also helpful for studying the urethra [117]. However, further studies on this application are needed. Voiding urosonography can be considered the method of choice for the initial study of suspected VUR in girls, in whom information about the urethra is less important, whereas voiding cystourethrography continues to be the first choice in boys, though voiding urosonography also shows potential in this setting [117]. Conventional cystography remains the reference technique in patients with abnormal urethral findings and/or to plan surgery.

\subsection{Limitations}

Voiding urosonography is not recommended as the primary imaging modality for reflux, if the bladder or one of the kidneys is not depicted on ultrasound and for specific urethral and/or bladder functional and anatomical evaluation [113].

\section{Recommended uses and indications}

Voiding urosonography is recommended in the following clinical situations:

1. First examination for vesicoureteral reflux in girls. (Recommendation Level: A;1a)

2. Follow-up examinations for vesicoureteral reflux in girls and boys after conservative or surgical therapy. (Recommendation Level: A;1a)

3. Screening high-risk patients for reflux (e.g. siblings, transplanted kidney). (Recommendation Level: A;1a)
4. The CEUS assessment of urethra in boys appears feasible and accurate, but not enough experience has been gained yet to recommend it in routine clinical practice. (Recommendation Level: 0;4)

\section{Scrotum}

\section{$\nabla$}

\subsection{Background}

Ultrasonography is the imaging modality of choice for examination of the scrotum. Occasionally, however, sonographic findings are equivocal and this may be important as a misinterpretation can result in an unnecessary orchiectomy. A particular conundrum is the unequivocal differentiation between hypovascular and avascular lesions, presuming that an avascular lesion implies benign disease: this may be impossible using colour Doppler US. CEUS provides a practical solution by increasing the confidence of the interpretation of lesion vascularity and of scrotal cord vessels, allowing for appropriate clinical management.

\subsection{Study procedure}

A full B-mode and colour Doppler examination of the lesion with linear high frequency transducers should be performed and recorded to relate to the subsequent CEUS findings. The arterial phase at CEUS is the most important aspect of the examination. The testis and epididymis enhance rapidly but the arrival time varies between individuals. The arteries enhance first, followed within seconds by complete parenchymal enhancement. The scrotal wall tends to enhance to a lesser degree than the contents. There is no accumulation of microbubbles in the parenchyma of the testis and the enhancement declines over a variable period of time such that there is minimal residual enhancement by 3 minutes.

\subsection{Patterns of disease}

\subsubsection{Torsion of the spermatic cord}

CEUS may be used as a problem solving technique in children with small testes where conventional Doppler imaging methods provide suboptimal assessment of flow. There is no firm data currently to inform the use of CEUS in spermatic cord torsion. In a small series of men with torsion, CEUS confirmed absence of vascularisation, but failed to add any clinically significant information to unenhanced colour Doppler US [118].

\subsubsection{Segmental Infarction}

The appearance of acute segmental testicular infarction on conventional B-mode and colour Doppler US is variable. Often the benign nature of the lesion is established by its wedge shape with markedly diminished or absent colour Doppler flow [119]. The main concern is the differentiation of a segmental infarct with a rounded configuration from a poorly vascularised tumour [120]. CEUS improves the characterisation of segmental infarction by showing one or more ischaemic parenchymal lobules separated by normal testicular vessels [121]. Subacute segmental infarction characteristically exhibits a perilesional rim of enhancement, which diminishes over time and is eventually lost with changes in lesion shape and shrinkage [121].

\subsubsection{Trauma}

Conventional B-mode and colour Doppler assessment of the testis in trauma is well established, but they under-estimate 
the extent of injury. Besides integrity or interruption of the tunica albuginea, the most important information for the surgeon is the extent of viable testicular tissue. CEUS adds to the colour Doppler assessment with clear delineation of fracture lines, intratesticular haematomas, and the amount of viable testis [122].

\subsubsection{Inflammation}

Epididymo-orchitis is a clinical diagnosis and is usually easily confirmed on colour Doppler US. CEUS may be able to determine the development of an abscess (appearing as a non-enhancing region) at an earlier stage, or the extent of a large abscess, and allow for prompt treatment [118]. CEUS may be utilised in selected cases to increase the sensitivity in detecting thrombosis of the spermatic cord vessels, especially in funiculitis, and in detecting post-inflammatory parenchymal ischaemic changes.

\subsubsection{Tumours and complex cysts}

The current understanding is that testicular tumours with a diameter of less than $1.5 \mathrm{~cm}$ may not show flow on colour Doppler US and thus may be misinterpreted as a benign lesion, the purported hallmark of malignancy being an increase in vascularity. Simple testicular cysts are usually benign, but any wall irregularity or echogenic debris may be suggestive of a (rare) cystic testicular tumour $[123,124]$. It is thought that virtually all testicular tumours display vascularisation on CEUS, with the exception of any cystic component and regions of necrosis [125]. Experience suggests that CEUS is able to confirm the absence of vascularity in benign complex and epidermoid cysts [126]. There is currently no evidence in the literature with regards to these findings.

\section{Recommended uses and indications}

CEUS for the testis can be currently recommended for:

1. Discrimination of focal testicular lesions into those that are vascular and those that show no enhancement, potentially identifying lesions without malignant potential. (Recommendation Level: $\mathrm{B} ; 2 \mathrm{~b}$ )

2. Discrimination of areas of non-viable tissue in cases of testicular trauma. (Recommendation Level: B;4)

3. Detection and characterisation of segmental infarction. (Recommendation Level: $\mathrm{B} ; 2 \mathrm{~b}$ )

4. Discrimination of abscess formation in severe epididymo-orchitis. (Recommendation Level: C;4)

\section{Abdominal Trauma}

\section{7}

\subsection{Background}

CECT is the modality of choice for the rapid detection and grading of neurological, skeletal, and thoraco-abdominal injuries in all cases of high-energy multitrauma. However, there is a wide range of severity among trauma patients who are admitted to an emergency unit, and the rate of positive findings on CT decreases with lower energy trauma. This should be weighed against the negative aspects of $\mathrm{CT}$, including exposure to ionizing radiation and injection of iodinated contrast media. US is sensitive in detecting free peritoneal fluid as an indirect sign of injury (Focused Assessment with Sonography in Trauma - FAST) but it has poor sensitivity in detecting solid organ trauma and is even less useful for hollow viscus trauma.
CEUS can increase the conspicuity of traumatic injuries, with a closer correlation with CT $[87,127,128]$. In a large, multicentre study [129], CEUS was more sensitive than US and provided sensitivities of $69 \%$ (renal), $84 \%$ (liver) and 93\% (spleen) compared to CT in the detection of solid organ injuries with very high specificity (over 90\%).

\subsection{Study procedure}

Contrast enhancement lasts long enough to allow an adequate exploration of all parenchymal organs in the trauma patient. The kidneys have the earliest and most transient enhancement and the spleen is inhomogeneous initially and then becomes persistently and homogeneously enhanced. Consequently the examination sequence usually starts with the kidneys, then moves to the liver, and finally examines the spleen. An alternative is to split the contrast dose in two, using the first to explore the right kidney and then the liver, and the second for the left kidney and the spleen. However, in many instances the CEUS study directly targets the organ suspected or known to be injured.

\subsection{Image interpretation of abdominal injuries}

CEUS demonstrates the injuries as non-enhancing defects, sharply demarcated from the intensely enhanced, undamaged tissue, particularly during the venous phase. Contusion appears as an ill-defined area of subtle hypoenhancement. Lacerations are depicted as markedly hypoechoic bands, usually oriented perpendicular to the organ surface; sometimes the torn organ capsule is seen as a discontinuity. A haematoma is recognisable as a non-enhancing area without internal enhanced vessels. CEUS can detect changes that are not apparent on conventional US, such as infarcts, perilesional hyperaemia, pseudoaneurysm formation, as well as contrast extravasation, which appears as a pooling or as a jet of contrast enhancement outside blood vessels. The spread of contrast enhanced blood into the parenchyma, the peritoneal cavity, or the retroperitoneum is an important finding since it indicates on-going haemorrhage and the need for surgery. As shown both by experimental and clinical studies, CEUS can detect ongoing bleeding from a variety of traumatic and also non-traumatic sources [87].

Emergency CT scans of trauma patients can have artefacts and CEUS may be useful for better assessing unclear findings. Furthermore, CEUS may be used in patients not ideal for CECT, such as children (see section 7). CEUS may decrease the number of repeat US examinations and expedite patient discharge. Several published series have demonstrated that CEUS allows a confident exclusion of major abdominal injuries. Hence, many patients with minor trauma can be discharged after a period of observation, without being submitted to CT at all.

\subsection{Limitations}

Since UCAs are not excreted in the urine, CEUS cannot be used for detecting damage to the urinary excretory system.

\section{Recommended uses and indications}

CEUS can be employed consistently in trauma imaging, considering the wide spectrum of scenarios encountered in clinical practice.

1. As an alternative to $\mathrm{CT}$ in stable patients with isolated blunt moderate-energy abdominal trauma to rule out solid organ injuries, especially in children (Recommendation Level: A;1b) 
2. To further evaluate uncertain CT findings (Recommendation Level: $\mathrm{C} ; 2 \mathrm{~b}$ )

3. For the follow-up of trauma managed conservatively, because it may make it possible to reduce in the number of CT scans or increase confidence when the situation does not strictly require a CT scan. (Recommendation Level: B;1b)

\section{Lung and Pleural Lesions}

\section{$\checkmark$}

\subsection{Background}

US imaging of the healthy lung is impossible because the US beam is totally reflected at the lung surface. Consolidations can be visualised when they reach the pleura, are accessible via an acoustic window, and there is no subcutaneous emphysema or pneumothorax [130]. CEUS assessment of the lung is therefore addressed to lung consolidations.

Because of its dual arterial supply, the lung has similarities to the liver and CEUS allows the differentiation of the pulmonary from the systemic bronchial arterial supply of consolidations by assessing the timing and extent of enhancement [131].

\subsection{Study procedure}

For lung consolidation, a conventional convex abdominal transducer is most commonly used. Before injecting the UCA, the best probe position to visualise the consolidation should be determined using B-mode and colour Doppler US and then observed for at least the first $30 \mathrm{~s}$ after contrast administration [132].

\subsection{Image interpretation}

Contrast arrives in the right heart between 1 and $5 \mathrm{sec}$ after injection and immediately thereafter enters the pulmonary artery (indicating the beginning of the time window of pulmonary arterial enhancement), and between 8 and $11 \mathrm{sec}$ enters the left heart (determining the start of systemic bronchial arterial enhancement immediately thereafter) in healthy subjects. An arrival time in the consolidation under study of less than 10 seconds ("early arterial enhancement") indicates pulmonary arterial supply and a delayed arrival time over 10 seconds ("late arterial enhancement") indicates supply by the bronchial arteries. In patients with cardiac and/or pulmonary disease, the arrival time for pulmonary arterial supply may be longer than 10 seconds [131].

\subsubsection{Pneumonia}

Contrast arrives early in pneumonia $(10 \mathrm{sec})$ and enhancement is marked [131] followed by a plateau caused by a combined supply from pulmonary and bronchial arteries [132]. In addition, CEUS allows demarcation of necrosis or abscess formations in the infiltrated lung tissue as regions of absent enhancement [131].

\subsubsection{Pulmonary embolism}

In embolic pulmonary infarcts, the arrival time is delayed and the degree of enhancement is minimal [131] within the first 30 seconds after UCA administration [132]. Such a decrease in enhancement is most evident early after pulmonary embolism and progressively recovers, so that infarctions are more clearly recognisable early after their onset. Quantification of contrast enhancement using dedicated software may provide more consistent information. Pleurisy or pneumonia can be clearly differentiated from pulmonary embolism by early and intense pulmonary artery enhancement [131-133].

\subsubsection{Pulmonary carcinoma and metastasis}

The value of CEUS for lung cancer is the improved delineation of viable from non-viable tissue before biopsy compared to colour Doppler US [134]. Lung tumours may behave very variably as regards contrast distribution on CEUS, but on average the time of arrival is late arterial as the arterial supply is from the bronchial system and not from pulmonary arteries $[131,132]$.

\subsubsection{Atelectasis}

In atelectasis, the CEUS pattern is similar to pneumonia with early and marked enhancement $(<10 \mathrm{sec})$ followed by a plateau [132]. An underlying central tumour may be better differentiated from the atelectasis by late enhancement [134].

\section{Recommended uses and applications (applicable only to} peripheral lesions visible on US)

1. CEUS is useful in differentiating inflammatory from embolic lung consolidation, especially in patients with equivocal CT findings. (Recommendation Level B;2b)

2. CEUS may be used in equivocal cases to diagnose lung abscesses within pneumonia, since CEUS appears better than conventional B-mode US or X-ray. (Recommendation Level C;5)

\section{Vascular}

\subsection{Background}

There are two major extracerebral vascular systems with common indications for CEUS. One is the cervical carotid artery and the other the abdominal aorta. Conventional US techniques are limited in demonstrating slow flow, especially in small vessels such as vasa vasorum or collaterals and flow in critical stenoses. UCAs help to overcome some of these limitations and might be used also in other extracerebral vascular districts, but such usage is less well established and is not reported herein.

\subsection{Study Procedures}

As for conventional investigations, CEUS of the carotid artery is carried out with linear probes working in the range of $5-10 \mathrm{MHz}$ and the abdominal aorta with convex probes at $2.5-5 \mathrm{MHz}$.

\subsection{Carotid artery}

\subsubsection{Stenosis}

Duplex US is the first line imaging modality for suspected carotid artery disease [135-138]. CEUS improves the sensitivity of Doppler US and can be used for distinguishing occlusion from tight sub-occlusive stenosis, even with conventional US equipment. However, when a contrast-specific modality is available, low MI CEUS can be utilised to further improve the delineation of the endovascular border in difficult cases, making it possible to detect the shape of pre-stenotic, intra-stenotic and post-stenotic segments, especially in elongated vessels $[139,140]$. This is also facilitated by the fact that high velocity and low-velocity flow phenomena are registered at the same time with CEUS without aliasing and blooming artefacts and without any angle dependence, which all affect Doppler US 
$[139,140]$. However, CEUS does not provide flow information, which may still be obtained by Doppler US, potentially facilitated by contrast injection. Plaque ulceration can be imaged using CEUS [141].

\subsubsection{Follow-up after carotid stenting}

CEUS is a reliable method for evaluating re-stenosis after internal carotid artery stenting [142]. CEUS has fewer intrastenotic flow artefacts compared to colour and power Doppler US, resulting in better visualisation and detection of the complete length and morphology of the stenosis [142].

\subsubsection{Dissection}

The pathognomonic imaging findings are mural haematomas and the depiction of a true and false lumen and small flaps. Blood flow is usually decreased in the false lumen [143], increasing the risk of thrombosis, eventually resulting in complete occlusion or emboli [144]. MRI is the reference standard in the diagnosis of cervical vessel dissections; when it is contraindicated, the diagnostic accuracy of US examinations can be improved by the use of CEUS [139, 140].

\subsubsection{Complications after vascular intervention}

Due to aliasing artefacts, correct visualisation of post-surgical fistula tracks can be difficult using conventional colour Doppler US. However, with CEUS, fistula tracks can be detected without aliasing or overwriting artefacts [145]. Additionally, CEUS may help to image flow in false aneurysms with greater precision than colour Doppler US.

\subsubsection{Plaque characterisation}

The most widely accepted predictor of stroke risk is the degree of carotid stenosis. However, other imaging features are becoming recognised as significant factors [146]. Plaque neovascularisation demonstrated by CEUS correlates well with histological findings [147, 148] and is a potential additional predictor of cerebral ischaemic events, best assessed by quantification software [141, 147-150], but any role in daily clinical practice is still to be demonstrated.

\subsection{Abdominal aortic aneurysm (AAA)}

CEUS can overcome some limitations of conventional US by improving the delineation of the aortic lumen and the detection of the main branching arteries. CEUS also improves the diagnosis of aortic rupture by detecting contrast extravasation [151].

\subsubsection{Aortic dissections}

Usually dissection of the abdominal aorta is an extension of thoracic aortic dissection [151]. Up to $38 \%$ of aortic dissections are missed on initial imaging examination and up to $28 \%$ of aortic dissections remain undetected until autopsy [152155]. In most cases, the true and false lumen can be discriminated with CEUS, because both early (true lumen) and late (false lumen) contrast enhancement can be detected, provided the false lumen is not thrombosed $[153,155]$. Vascular complications such as renal or splenic infarction can be evaluated [139].

\subsubsection{Inflammatory Abdominal Aortic Aneurysm}

Inflammatory aneurysm of the abdominal aorta is a variant of atherosclerotic aneurysm that is characterized by inflammatory and/or fibrotic changes in the periaortic regions of the retroperitoneum $[156,157]$. The triad of a thickened aneurysmal wall, extensive perianeurysmal and retroperitoneal fibrosis, with adhesions to adjacent abdominal organs, should suggest this diagnosis [158-160]. Contrast enhancement in the inflammatory aneurysm improves differentiation between covered aortic rupture and inflammatory aneurysm [161].

\subsubsection{Endoleaks}

Early and midterm results of endovascular repair of AAA are promising but long-term durability and outcomes of this technique are still unclear. Endoleaks represent blood flow outside the stent graft lumen but within the aneurysm sac and are conventionally detected by CT angiography, which however, does not allow detection of some types of endoleak [162]. Several authors have pointed out the usefulness of CEUS because it seems to identify and characterise endoleaks better than CT angiography, with analysis of velocity and flow direction [163-169]. CEUS enhancement quantification by TIC provides additional accuracy [168]. Once an aortic endoleak has been detected, CEUS can be used for the follow-up of patients [170].

\subsection{Limitations}

Limitations in the CEUS exploration of the carotid artery and abdominal aorta are any conditions which prevent adequate US penetration and which also limit conventional B-mode US exploration. Of particular regard are extensive wall calcification and subcutaneous emphysema after intervention or limited examination windows.

\section{Recommended uses and indications}

CEUS is recommended for the following indications:

1. Differentiation between total carotid occlusion and residual flow in tight stenoses by contrast enhanced Doppler or CEUS. (Recommendation Level: $\mathrm{B} ; 3$ )

2. Improvement of lumen delineation in technically difficult carotid arteries (contrast enhanced Doppler US can also provide useful information). (Recommendation Level: $\mathrm{B} ; 3$ )

3. Evaluation of carotid plaque neovascularisation. (Recommendation Level: $\mathrm{B} ; 1 \mathrm{~b}$ )

4. Improving the detection of dissection of the common and internal carotid arteries, vertebral artery and aorta. (Recommendation Level: $\mathrm{C} ; 3$ )

5. Detection of aortic wall rupture. (Recommendation Level: B;3)

6. As an additional tool in the characterisation of suspected inflammatory AAA. (Recommendation Level: C;5)

7. Detection and characterisation of endoleaks after AAA repair. (Recommendation Level: A;1a)

8. Follow-up of AAA endoleaks. (Recommendation Level: A;1a)

\section{Cerebral Vessels}

$\nabla$

\subsection{Background}

The major indication for CEUS in the examination of cerebral arteries is the poor signal-to-noise ratio using unenhanced Doppler US, which can prevent measurement of the Doppler traces for distinguishing no flow, slow flow or low flow [171]. Contrast enhanced transcranial colour-coded duplex sonography (CE-TCCS) is the best modality for simultaneously depicting B-mode brain anatomy as well as vessel detection and flow tracings. 


\subsection{Study procedures}

Transducers for CEUS are the same as for CE-TCCS, namely sector $1.5-4 \mathrm{MHz}$ probes. There are two applications using UCAs: main vessel, i.e. vascular imaging, and "perfusion imaging". For vascular imaging, UCAs are utilised to enhance Doppler signals in transtemporal or transnuchal transverse axial planes (insonation depth $10-12 \mathrm{~cm}$ ). Coronal transtemporal planes may also be used. In order to obtain adequate information, some technical artefacts should be considered: 1 ) bolus injection may cause blooming artefacts preventing accurate Doppler spectral measurements. This artefact can be reduced by reducing the total gain or by slow infusion of the UCA (1-2 mL SonoVue ${ }^{\circledast} / \mathrm{min}-$ ute). 2) UCA injection leads to an artificial increase (15-36\%) in maximum blood flow velocity [172]. This may affect velocity criteria in the classification of stenoses. In perfusion imaging, either low or high MI CEUS is performed with transtemporal insonation in the axial plane with an insonation depth of 10 to $15 \mathrm{~cm}$. Other insonation planes may also be used.

\subsection{Main intracerebral vessel imaging: interpretation and evaluation \\ 18.3.1 Vascular imaging}

Most importantly, CE-TCCS is used to differentiate vessel occlusion in poor insonation conditions, and to detect of very slow blood flow velocities and low flow volumes (small vessels, vessel pseudo-occlusion). The Doppler spectrum adds haemodynamic information to the anatomical information provided by colour Doppler US.

\subsubsection{Examination of the anterior circulation}

A poor temporal bone window, as seen in up to $45 \%$ of elderly patients, can usually be overcome with CE-TCCS. In over $85 \%$ of such cases the basal arteries of the circle of Willis can be depicted satisfactorily after UCA administration [173]. According to expert opinion, CEUS with SonoVue ${ }^{\circledR}$ infusion can be used in patients with poor acoustic windows for transcranial Doppler monitoring to test cerebral autoregulation as well as language lateralisation for surgical planning.

\subsubsection{Examination of the posterior circulation}

CE-TCCS through the foramen magnum can increase the depth at which the intracranial vertebral arteries, the basilar artery, and the cerebellar artery segments can be identified and thus improve diagnostic confidence [172].

\subsubsection{UCAs in patients with internal carotid artery stenosis} Characterisation of flow in the circle of Willis in patients with internal carotid artery stenosis and poor bone windows is important for estimating the risk of ipsilateral border zone infarction. Patients without collateral flow are particularly vulnerable to cross-clamping during carotid endarterectomy. The use of UCA in these patients can provide valuable information for patient management [174].

\subsubsection{UCAs in stroke patients}

In acute stroke, the basal cerebral arteries can only be detected in $55-80 \%$ of cases with unenhanced TCCS. Fortunately, reliable diagnoses can be obtained in more than $85 \%$ of these patients after contrast enhancement, with correlative findings on angiography (MR, CTA or conventional angiography) in over $95 \%$ of cases [171].

\subsection{Perfusion imaging}

CEUS is performed with transtemporal insonation in the axial plane with an insonation depth of 10 to $15 \mathrm{~cm}$ in perfusion imaging. Other insonation planes may also be used. CEUS perfusion imaging has been shown to improve prognostic assessment in the acute phase and to provide comparable results to CT $[175,176]$ and MRI [177]. After bolus injection, TICs can be generated to extract features (time to peak intensity, peak intensity, area under the curve, etc.) that describe the perfusion characteristics quantitatively in standardised regions of interest $[171,178]$.

\subsection{Limitations}

Despite UCA administration, only the proximal basilar artery can be evaluated. The distal portion can be depicted transtemporally, leaving the middle portion as a diagnostic gap for CETCCS. The quality of transtemporal precontrast scans is strongly predictive of the potential diagnostic benefit from the administration of an UCA. In patients without visible intracranial structures and vessels on conventional B-mode and colour Doppler US, there is little chance that CEUS will provide satisfactory diagnostic information.

The combination of systemic thrombolysis and repeated administration of a UCA over an hour in patients with middle cerebral artery occlusion accelerates recanalisation but also increases haemorrhage into the infarct [179]. While of greatest interest as a way to enhance therapy (which has not yet been fully evaluated) this is a potential risk when repeated CEUS studies are performed.

The clinical value of the quantification of enhancement is limited by both physical and technical factors. Up to now, they can only reliably characterise the absence of enhancement rather than the exact degree of blood supply impairment.

\section{Recommended uses and indications}

1. Contrast enhanced transcranial colour duplex sonography can be diagnostic in patients with unsatisfactory intracerebral information with non-enhanced transcranial colour duplex sonography. (Recommendation Level: A:1b).

2. CEUS perfusion imaging based on quantification of brain enhancement provides prognostic information in acute stroke. (Recommendation Level: C;4).

\section{Inflammatory Joint Diseases}

\section{$\nabla$}

\subsection{Background}

Colour/power Doppler US can detect the vascularity in synovial proliferation reflecting inflammatory activity. However, these techniques have limited sensitivity and they benefit from the addition of UCAs.

\subsection{Study procedure}

Reports on the use of UCAs in inflammatory joint disease are mainly about the enhancement of signals detected by conventional colour/power Doppler US (CE Doppler US), rather than by using low MI CEUS. The standard probes and equipment used for the second level investigation of joints are also utilised for CEUS. 


\subsection{Image interpretation}

\subsubsection{Arthritis and synovitis}

Microscopic examination of synovial biopsies shows angiogenesis from the earliest stages of inflammatory disease. Proliferation of hypervascularised pannus can be detected before joint destruction. It correlates with disease activity and appears to be crucial to its invasive and destructive behaviour [180]. Moreover, the development of novel biological therapies (e.g. tumour necrosis factor-alpha inhibitors), which target the microvasculature, have created a demand for more sensitive vascular imaging in order to assess response to treatment [180184]. The addition of UCAs to Doppler US significantly improves the detection of vascularity in active rheumatoid arthritis $[185,186]$. Following CE Doppler US, therapy was modified in up to $24 \%$ of patients in a dedicated study [185]. There is also evidence that CE power Doppler US helps to differentiate between active and inactive disease in subclinical juvenile rheumatoid arthritis of the knee [187]. CE Doppler US correlates with the findings of CEMRI, indicating the degree of inflammation in patients with synovitis [188]. CE Doppler US is also more useful in the diagnosis of sacro-iliitis than conventional unenhanced Doppler US [182, 189]. Overall, improved diagnostic accuracy in assessing the degree of inflammation can be achieved by the addition of UCA in large and small joints.

\subsubsection{Differentiation between synovial pannus and fluid}

MRI studies have demonstrated that the presence and amount of synovitis is a prognostic factor for bone damage [190]: no bone damage occurs in joints without synovitis. Therefore, early detection of vascularised synovia is a primary goal in assessment of inflammation. CE Doppler US improves the differentiation between active synovitis and other articular thickenings such as fibrotic pannus and articular fluid [191, 192].

\subsubsection{Bursae and tendons}

Contrast administration can highlight peripheral enhancement on Doppler US corresponding to the vascularised synovial lining of an inflamed bursa and can better differentiate between fluid, fibrous and hypervascular synovial thickening in comparison to non-enhanced Doppler US [193, 194].

\subsubsection{Therapeutic follow-up}

Successful treatment results in a decrease in synovial thickening and necrosis of the pannus with reduction of vascularity and thus of Doppler signals. The distinction between fibrous pannus and active synovial proliferation is one of the most important questions during follow-up, because the volume of the synovium itself is not clinically significant, as it may contain varying amounts of fibrous tissue [190]. On unenhanced power Doppler US, fibrotic pannus shows no vascularity as also after addition of UCA into fibrotic pannus shows no vascularity, as it also does after the addition of UCA and, with CEUS, lacks enhancement [195-199].

\section{Recommended uses and indications}

Contrast enhanced colour/power Doppler US:

1. Can be utilised for further assessment of the degree of vascularisation in joints of patients with rheumatoid arthritis. (Recommendation Level: $\mathrm{C} ; 2 \mathrm{~b}$ )

2. Repeat contrast enhanced Doppler US may provide useful information on the response to treatment, to guide therapeutic strategy. The technique has the potential to be utilised within dedicated centres using standardised methodology. (Recommendation Level: $\mathrm{C} ; 2 \mathrm{~b}$ )

\section{Intracavitary Uses}

$\nabla$

\subsection{Introduction}

Reports on numerous off-label extravascular or intracavitary administrations of SonoVue ${ }^{\circledR}$ have been published and those considered of more general use and most consistent are reported. Other potential applications have been occasionally described as case reports and congress abstracts.

\subsection{Study procedure}

To date, no standard dosage of UCA has been established for intracavitary injection. The reported range is $0.1 \mathrm{~mL}-1 \mathrm{~mL}$ SonoVue ${ }^{\circledR}$ (most commonly just a few drops) diluted in $10 \mathrm{~mL}$ or more of $0.9 \%$ saline; a higher content of SonoVue ${ }^{\circledR}$ may be needed for high frequency US probes.

\subsection{Injection into Physiological Cavities}

\subsubsection{Voiding US for vesicoureteral reflux}

This topic is described in section 13.4.

\subsubsection{Imaging of tubal patency - contrast enhanced} hystero-salpingo-contrast-sonography (CE-HyCoSy)

Originally performed using shaken saline infused into the uterine cavity, hystero-salpingo-sonography suffers from a false negative rate of patency of about $12 \%$ [200]. CEUS with SonoVue ${ }^{\circledR}$ provides better results [200-202] with higher specificity, but the positive predictive value for the diagnosis of "occluded tube" remains low [200]. The high negative predictive value of conventional hystero-salpingo-contrast-sonography leads authors to suggest performing contrast enhanced hystero-salpingo-contrast-sonography only if conventional hystero-salpingocontrast-sonography does not show patency.

\subsubsection{Detection of peritoneo-pleural communciation for the diagnosis of hepatic hydrothorax}

The detection of direct communication between the abdominal and pleural cavities is the main indication reported so far $[203,204]$. Confirmation of the suspected diagnosis of hepatic hydrothorax can be established in cirrhotic patients by injecting SonoVue ${ }^{\circledR}$ into the peritoneal cavity, at best early $(<2$ days) after thoracentesis and demonstrating its passage into the pleural cavity.

\subsubsection{Bile ducts}

CEUS-guided percutaneous cholangiography was first described in 2009 [205]. A later case report on UCA injection into a surgically placed T-tube followed [206]. It is expert opinion that the advantage of CEUS-guided percutaneous cholangiography and drainage is that external drainage for biliary obstruction in patients with severe cholangiosepsis can be performed in the intensive care unit without X-ray guidance. UCA for endoscopic retrograde cholangiography has been reported [207].

\subsubsection{Salivary glands}

UCA injected into the main duct of a salivary gland could become a supplementary diagnostic method added to sialogra- 
phy to classify obstructive diseases of the salivary glands. The salivary gland is cannulated with appropriate dilatators and the plastic tube of a peripheral vein catheter is inserted [208].

\subsection{Injection into Non-Physiological Cavities \\ 20.4.1 CEUS for imaging of fistula}

CEUS detection and classification of fistulas, whatever the initial disease, has been proven to be effective. The following conditions have been reported: rectovaginal fistulas via a transvaginal approach [77], vesicointestinal fistulas via a transabdominal approach [209] and anal fistulas via the transrectal approach [210].

\subsection{Other intracavitary indications for CEUS}

In principle, UCAs can be instilled into any sonographically accessible physiological or pathological body cavity to assess morphology of the cavity and potential communications with adjacent structures or organs. Clinical applications include: abscesses, pancreatic pseudocysts and other complications of pancreatitis, intestinal and other fistulas, gastro-oesophageal reflux, gastric lumen and intestinal stenoses.

\section{Recommended uses and indications}

No definite recommendation can be made for these indications. Use of CEUS must be decided on a case-by-case basis, usually resorting to CEUS because of inconclusive standard diagnostic techniques or when patients are not suitable for these or because they have higher risks (for instance, use of ionizing radiation on sensitive organs, or moving the patient out of intensive care units) or more complex logistic situations.

\section{Lymph Nodes}

\section{$\nabla$}

\subsection{Background}

The differential diagnosis of superficial lymphadenopathy in cancer patients has been the object of various studies with high-resolution US. Their shape, appearance and vascular pattern have been analysed to discriminate benign from malignant nodes, with a wide range of sensitivities and specificities $[211,212]$. CEUS has been shown to increase the accuracy of the analysis of the vascular pattern (angioarchitecture) [213]. Contrast enhanced investigations were first performed with high MI US using Doppler and later with low MI CEUS.

\subsection{Study procedure}

The same probes as for conventional B-mode US are utilised but with contrast-specific modalities and a full vial of SonoVue $^{\circledR}$, as for other superficial applications.

\subsection{Image interpretation and differential diagnosis of benign and malignant lymph nodes}

Demonstration of malignant neovascularisation, seen as vessels penetrating the node's capsule away from the hilum, has been used in most reports as the characteristic feature of metastatic lymph nodes. Confirmation of the reactive nature of lymph nodes relies on their preserved morphology and vascular anatomy, with a single vascular pedicle at the hilum containing arteries and veins vessels, regularly branching towards the periphery of the lymph node. Display of vascular anatomy can be facilitated by the addition of UCAs during colour Doppler US. Conventional CEUS using low MI can improve on the results of Doppler US with a sensitivity, specificity and accuracy rate of up to $84 \%, 79 \%$ and $80 \%$ [214-223].

However, the US study of lymph node vascularisation has limitations. Most studies were made in special clinical settings (known head and neck or gynaecologic cancer). Vessel distribution analysis is usually satisfactorily informative if the whole lymph node is involved. This is not always the case, because of the possibility of focal cancer metastases or necrosis. Lymphoma must be considered separately because there is evidence that the vascular pattern of lymphoma lymph nodes resembles that of non-malignant nodes [224, 225].

\subsection{Sentinel lymph nodes}

CEUS can be used for detecting sentinel lymph nodes in cancer patients. One $\mathrm{mL}$ of SonoVue ${ }^{\circledR}$ is injected subcutaneously near the tumour site and the enhanced lymphatics are traced to the sentinel node [226-231]. Initial experiences indicates that the method is not toxic and performs as well as the blue dye or radioisotope methods [231].

\section{Recommended uses and indications}

1. CEUS appears to be capable of discriminating benign from malignant superficial lymph nodes only in special clinical settings. Therefore, despite some positive publications, it is the opinion of the experts that CEUS is not recommended for routine discrimination of benign from malignant lymph nodes. (Recommendation Level: $\mathrm{C} ; 5$ )

2. CEUS with subcutaneous injection of contrast agent to identify the sentinel lymph node is a field of ongoing research and can therefore not be recommended for clinical practice to date. (Recommendation Level: C;5)

\section{Tumour Response Assessment \\ $\nabla$}

\subsection{Background}

The advent of novel therapies targeting tumour angiogenesis and vascularity has highlighted the need for accurate and reproducible quantitative techniques to assess early changes in tumour vascularity [232]. However, as these therapies are predominantly cytostatic, current response assessment which is based on interval evaluation of the tumour size using the Response Evaluation Criteria In Solid Tumours (RECIST) [233] is inadequate as it reflects only late changes and is unable to identify non-responders at an early time-point [234].

\subsection{Study procedure}

Dynamic contrast enhanced US (DCE-US) can be performed using two different approaches with different results and evidence produced so far:

(a) bolus injection of UCA with TIC analysis; single plane imaging is usually performed at 10-20 frames per second for the duration of the enhancement. The average intensity within a region of interest (ROI) can be displayed as a function of time, i.e. a TIC which describes the wash-in and wash-out of the UCA in the ROI [235]. In addition, a second ROI can be placed in a reference tissue for comparison purposes [236]. The majority of clinical studies to date are based on this method.

(b) intravenous infusion of UCA with disruption-replenishment analysis. The UCA is administered using a pump or drip over 5 to 20 minutes. UCA is first imaged without being disrupted at a low MI, then the MI is increased for a few frames, causing 
bubble disruption. Immediately after that, the MI is returned to the non-disrupting level to observe the replenishment of the microbubbles into the ROI. Various models describe the echo-signal dynamics during the UCA-replenishment phase, which can be used for flow analysis [237].

Initially monitoring for tumour response with UCAs relied on qualitative analyses [238], but new methodologies have been developed to produce more robust and semi-quantitative indices. Analyses of the TIC, including wash-in and wash-out times, can be performed with curve fitting to determine functional indices [239]. The main indices include: peak intensity (PI); area under the curve (AUC); area under the wash-in (AUWI); area under the wash-out (AUWO; all corresponding to blood volume); time to peak intensity (TPI); slope of the wash-in (SWI; both corresponding to blood flow); and mean transit time (MTT). No permeability information can be obtained because of the pure blood pool nature of microbubbles. A more extensive EFSUMB introduction to quantitative enhancement indices is being prepared.

\subsection{Clinical application of DCE-US}

Early clinical trials employed qualitative analysis in the assessment of the response of different tumours such as gastrointestinal stromal tumour (GIST) or renal cell carcinoma [240-243]. More recently, there have been studies using semi-quantitative techniques with UCA bolus injection in renal cell carcinoma, hepatocellular carcinoma (HCC) and GIST [244-246]. Studies from the French group showed that two indices representing blood volume correlated with the RECIST response. For one study on renal cell carcinoma the authors demonstrated a correlation of such indices with Progression Free Survival and Overall Survival [244]. The same results could not be reproduced in a Canadian study using the disruption-replenishment technique tested versus Progression Free Survival assessed by the RECIST method [237].

A French multicentre study of various types of tumours, approximately half of which were located outside the liver (including more than 400 patients), such as metastatic renal cell carcinoma, GIST, colon cancer, melanoma, breast cancer and HCC, treated with anti-angiogenic therapies has been running, but not yet reported in its complete form. The preliminary results found that the AUC is one of the features correlated to response at 6 months in good and poor responders [247], but the full results of the data set are not yet available. There is now emerging evidence that DCE-US may be used with appropriate tools to differentiate between responders and non-responders at an earlier stage than conventional methods and this potentially allows tailoring of the treatment regimen, particularly changing treatment for non-responders. DCE-US has been endorsed by the European Medical Oncology Society to assess response under biological therapy for GIST [248].

\section{Recommended uses and indications}

1. DCE-US can be utilised to assess response to biologic therapy in metastatic GIST and other metastatic tumours, e.g. renal cell carcinoma, in dedicated centres with appropriate software for contrast signal quantification. (Recommendation Level: A;1b)

\section{Breast}

$\nabla$

The application of CEUS to the differential diagnosis of breast masses was one of the first [249] and the initial results were very encouraging. Sadly, this early promise has not been fulfilled, despite numerous studies using modern methods [250] (summarised in [251]) including temporal accumulation methods (microvascular imaging) [252]. No specific pattern indicating malignancy has been identified. Thus, CEUS of the breast remains an important topic for research but cannot be recommended for routine clinical use. Among the different fields of research, that on contrast quantification appears the most promising [253].

\section{Adrenal Glands}

$\nabla$

So far, no CEUS criteria that can reliably differentiate between benign (endocrine tumours and adenomas) and malignant adrenal masses are known and indeed, the specificity of CEUS in the diagnosis of malignant adrenal masses was reported to be below 70\% [254]. Malignant adrenal tumours may infiltrate and occlude the adrenal vein. The vascularity of a tumour thrombus can be shown by UCA and thus its charactar be demonstrated.

CEUS may demonstrate characteristic hypervascularity of some adrenal gland tumours, e.g. pheochromocytoma, which typically also have necrotic regions with no contrast enhancement [255].

\section{Emerging Perspectives and Potential Future Applications for CEUS}

$\nabla$

\section{A. Obstetrics and Gynaecology}

\section{A. 1 Obstetrics}

The use of UCAs in obstetrics is not indicated because of lack of testing and underlying fears of toxicity. There is older literature in humans on the topic but none that is recent in animal models. It is unknown whether the microbubbles pass through the placenta, though this seems unlikely. CEUS to assess a pregnant mother should be balanced against the risk of other modalities. The opportunities seem very limited and the subject is not considered further here.

\section{A.2 Gynaecology}

\section{A.2.1 Uterus}

Research has included uterine and cervical tumours [256], flow differences between endometrial polyps and cancer [257], and CEUS during uterine artery embolisation to treat leiomyomas [258]. So far, no prospective trials confirm the value of CEUS to assess uterine tumours and thus there is no proven clinical indication for its use in examining the endometrium or the myometrium.

\section{A.2.2 Adnexa}

Differentiation of benign from malignant adnexal masses was attempted by visual assessment of contrast distribution and by quantification of enhanced Doppler signals but, despite some difference in average values for some variables, no feature with sufficient clinical potential was obtained [259]. 
By using CEUS it was demonstrated that adnexal masses without internal enhancement are invariably benign, and this feature was detected with high intra- and interobserver reproducibility [258], but the presence of enhancement is not a specific sign of malignancy [256]. CEUS does not greatly improve the accuracy of colour Doppler US for the diagnosis of malignancy in adnexal masses [260]. A large multicentre study on the diagnosis of malignancy in adnexal masses, including quantitative CEUS features, confirmed that CEUS is not superior to conventional US, despite being statistically more accurate [260]. Both also have difficulty in distinguishing between benign and borderline tumours.

At present, there are no recommended gynaecological clinical indications for the use of CEUS, despite the finding that absence of any enhancement in adnexal masses corresponds to benign lesions. (Recommendation Level: $A ; 2 b$ ).

\section{B. Perineum}

CE perineal US is an effective, easily available but so far not well known diagnostic tool. Perineal US is particularly useful if clinical examination, endorectal US or MRI with endorectal coils cannot be performed (e.g., due to severe pain when introducing the probe). CE perineal US requires good knowledge of the anatomy of the pelvic floor and the sphincters. No patient preparation is required. The location of inflammatory and neoplastic lesions should be described in relation to the sphincter apparatus. Fistulas can be further differentiated by US examination into intersphincteric, transsphincteric and extrasphincteric forms. Conventional B-mode perineal US is inadequate for complex fistulas, as its sensitivity for the complex branching structure is poor. However, discrimination of fistulas and abscesses with CE perineal US is possible in most cases, but studies have not been published so far. Additionally, the extent of a fistula can be imaged by instillation of UCA into its external ostium. CE Doppler US has shown a higher resistance index (RI) in neoplasia compared to inflammation, but further studies are needed.

\section{C. Urinary Bladder \\ 25C. 1 Background}

In patients with urinary bladder tumours, noninvasive diagnostic imaging may play a role, even though it cannot replace cystoscopy and pathologic staging. The depth of wall invasion, as well as the histological grade and the extension outside the bladder are the three main factors determining prognosis and therapeutic approach.

\section{C.2 Study procedure}

Optimal bladder filling (approximately two thirds of the total bladder volume) is critical [261]. Insufficient filling prevents lesion detection, while excessive distension results in bladder wall thinning and reduced conspicuity of the wall layer and may make it more difficult to differentiate a superficial tumour from an infiltrating lesion.

The layers of the bladder wall can be differentiated after microbubble injection. The mucosa, and especially the submucosal layer, exhibit early and intense enhancement that persists for 1-2 minutes. The muscular layer has lesser and delayed enhancement [261].

\section{C.3 Image interpretation}

\section{C.3.1 Characterisation of mural masses}

CEUS improves the differential diagnosis of intraluminal echoic masses, allowing the detection of tumours, which are vascularised and thus enhance [262], while clots do not enhance [263]. In a group of 35 patients in whom cystoscopic biopsy was the reference standard, CEUS correctly assessed the presence or absence of tumour in $88 \%$ of cases [263].

\section{C.3.2 Bladder tumour staging}

CEUS is superior to conventional B-mode US in identifying infiltration of the muscle layer [261], but MRI and CT are the imaging modalities of choice for local staging of bladder tumours. Attempts to predict tumour grading by the pattern of CEUS enhancement is still a research topic [262].

\section{C.4 Limitations}

Similarly to other imaging modalities, the most important limitation of CEUS in bladder tumour detection is the difficulty in identifying subcentimetre lesions. Flat, plaque-like tumours may also be difficult to detect, even when large. Tumour position can affect the quality of depiction at CEUS, and therefore the accuracy of staging. Tumours in the anterior portion of the bladder dome are sometimes difficult to visualise. Columnar hypertrophy of the bladder wall associated with benign prostate hypertrophy can hide or mimic urothelial polypoid projections, as well as prostate hypertrophy itself [261]. Benign tumours and focal cystitis are other uncommon conditions that present with focal bladder wall enhancement and can mimic a malignant lesion. Finally, CEUS is not panoramic as CT and MR are.

To summarize, the most useful application of CEUS is the differential diagnosis of bladder cancer from clots in patients with haematuria when the diagnosis is equivocal on conventional B-mode and Doppler US. (Recommendation Level: C;2b). In patients with anatomical situations leading to poor urinary bladder visualisation, CEUS often does not provide the desired information. (Recommendation Level: X;5).

\section{D. Transplanted Kidney}

Progressive vascular remodelling in the transplanted kidney, from a variety of mechanisms, compromises renal perfusion and accounts for the majority of allograft failures. CEUS has the potential to investigate perfusion abnormalities in renal transplants and to obtain information on blood flow based on the quantification of contrast enhancement or on the depiction of devascularised regions (the latter with the same significance as in native kidneys, so that CEUS use for this indication can be considered as established also in transplanted kidneys). Different quantitative features have been assessed, all related to impaired parenchymal perfusion (e.g. longer time to peak, lower wash-in slopes, longer mean-transit time) and associated with a worse prognosis of graft function and survival [264].

Although these preliminary results are promising, further studies are needed to assess whether detecting haemodynamic changes in renal grafts affects the management of patients with poorly functioning transplants; consequently the quantification of CEUS is still considered a research field in transplant assessment. 


\section{E. Prostate Cancer}

Conventional B-mode and Doppler transrectal US imaging have a limited role in the detection of prostate cancer because of poor sensitivity and specificity (approximately 50-60\%). However, there is a correlation between microvascular density and the presence of prostate cancer, its stage and survival. Therefore, attempts were made with CE transrectal US with colourDoppler to improve the detection of additional cancer nodules; with this modality the detection rate of targeted biopsies increased by almost $50 \%$ compared to systematic biopsies in some studies [265, 266]. Low MI transrectal CEUS became available only recently when contrast specific modalities were implemented on endocavitary probes. Preliminary single centre results appeared to confirm the findings of CE Doppler US, despite the lack of specificity of enhancing areas and of any other pattern [267-269]. The evidence achieved so far with CEUS modalities is, however, still too limited and not sufficiently confirmed in clinical practice in other centres, so that the role of CEUS in prostate cancer should still be considered a research topic, with hopes of improvements as new techniques are becoming available, such as 4D contrast enhanced transrectal US imaging.

\section{F. Aorto-Caval Fistula}

Perioperative mortality of abdominal aortal aneurysms complicated by aorto-caval fistulas undergoing open surgery is up to $60 \%$ and the additional retroperitoneal rupture of the aneurysm considerably raises the mortality [270]. The diagnosis can be made by noninvasive testing, such as US scanning, including CEUS, which improves visualisation of the fistula track and of the number of fistulas, avoiding the aliasing or the overwriting artefacts typical of Doppler US.

\section{G Free Tissue Transplants}

\section{G.1. Background}

Free flap reconstruction of complex defects after trauma, tumour resection, burns, or wound healing disturbances has revolutionized plastic, reconstructive, and microsurgery, giving the unprecedented potential to provide form, function, and volume for defects in every body region. However, despite refinements in this technique over the last several decades that have resulted in a decreased rate of flap loss of less than 5 $10 \%$, in general, the loss of free flaps because of vascular impairment remains a serious complication. Early identification of vascular deterioration and prompt revision could save up to $33-57 \%$ of failing flaps. CEUS offers the possibility of earlier detection [271-273].

\section{G.2 Study procedure}

Standard doses (2.4-4.8 mL of SonoVue ${ }^{\circledR}$ ) and administration methods are used together with high frequency probes $(\geq 6 \mathrm{MHz})$ to evaluate the microcirculation of the cutaneous, subcutaneous, and deeper layers of free flaps. Beside the standard visual evaluation, improved diagnostic assessment is obtained by contrast specific software working on the acquired information, including the production of TIC in different tissue layers by demarcation of different ROIs to quantify enhancement (274). Postoperative TIC analysis allows calculation of peak and time to peak of enhancement and regional blood volume. Exported cine-loops allow colour-coded depiction of these features with dedicated software $[275,276]$.

\section{G.3 Image Interpretation}

25G.3.1 Preoperative planning of tissue transplants

The blood vessels in the transplanted free flap are small (at most $1-2 \mathrm{~mm}$ ). The surgeon needs to know the precise number, course and position of these blood vessels as well as the flow in order to estimate the proportion with a good blood supply.

\section{G.3.2 Intraoperative imaging}

CEUS enables the identification of perforator vessels intraoperatively, and helps to determine if there are any abnormalities. The surgeon can thus make a more accurate decision as to whether the entire flap is perfused and if the estimated flap size is correct.

\section{G.3.3 Postoperative monitoring of tissue transplants}

The feeding vessels or, if there is a connection to a bypass, the anastomosis, and the transplant's own vessels can also be examined even if the vessel diameter is very small. CEUS can provide valuable information about a vascular compromise (thrombosis, embolism, twisting, kinking, or compression) and demonstrate successful surgical salvage [274, 277].

\section{G.3.4 Critical microvascularisation of free flaps}

A significant difference between normally vascularised and compromised flaps can be observed and the Time to Peak and Regional Blood Flow appear to be the most useful indices [278]. For CEUS and CEMRI, the mean signal increase of the TIC was significantly higher in ROIs of normally perfused flaps compared to compromised flaps [275, 276, 279, 280]. With CEUS, the exact size of the necrotic regions can be evaluated by analysing the entire flap. A haematoma or seroma can be detected early with CEUS, by the absent perfusion.

\section{G.4 Limitations}

The main limitation for the evaluation of flap perfusion is the fact that CEUS does not allow for continuous monitoring after bolus injection. Continuous infusion might be a better method, but remains to be explored.

To summarise, high-resolution CEUS is promising for confirming a clinical suspicion of malperfusion of free tissue transplants as well as for determining the cause. (Recommendation Level: $B ; 2 b)$.

\section{$25 \mathrm{H}$. Biliary disease}

\section{H. 1 Background}

The vascular phases of the gallbladder are different from those of the liver because the blood supply is provided entirely by the cystic artery and not by portal vein branches. The arterial phase is followed by the venous phase, which is shorter than that of the liver.

\section{H.2. Image interpretation}

\section{H.2.1 Acute cholecystitis}

In acute cholecystitis, the detection or exclusion of abscess formation in the surrounding liver parenchyma is important and can be performed with CEUS, but published evidence is sparse so far. Interruption of the gallbladder wall suggests perforation, which can be confirmed by the absence of enhancement of the perforated wall. 


\section{H.2.2 Extrahepatic biliary tumours}

The absence of enhancement in biliary sludge (whether in the gallbladder or in a dilated biliary tree) allows differentiation from a tumour, which enhances, in almost all cases using CEUS.

Gallbladder carcinomas are typically hyperenhancing in the arterial phase and hypoenhancing in the venous phase. The differentiation between benign and malignant lesions is mainly determined by clinical features and size and enlargment of polyps to $>10 \mathrm{~mm}$ is an indication for cholecystectomy. More sophisticated classification of the vascular and enhancement pattern of gallbladder lesions, including that provided by CEUS, has not been introduced so far in the clinical routine and CEUS currently has no role in differentiating benign from malignant gallbladder polyps.

The most important task of CEUS and other imaging methods is to detect infiltration of the surrounding liver parenchyma and to exclude liver metastases.

Transabdominal CEUS has no proven role when extrahepatic cholangiocarcinoma is suspected, although promising findings have been observed e.g., determining the depth of wall infiltration and surrounding tissue mainly by using CE-EUS. Intrabiliary administration of UCA is discussed in 20.3.4.

\section{Use of CEUS in Patients with Renal Failure \\ $\nabla$}

The use of iodinated/Gadolinium-containing contrast agents for CT and MR, respectively, potentially exposes patients with renal failure to severe complications [281, 282]. Assay of serum creatinine level and calculation of the estimated glomerular filtration rate before injection is mandatory in patients who may have impaired renal function (diabetes mellitus, age $>70$ years, history suggesting possible reduced eGFR, congestive heart failure, intensive diuretic treatment, dehydration, etc). Recommended guidelines to avoid contrast-induced nephrotoxicity from iodinated contrast agents [281] are available. However, none can guarantee completely successful prevention.

The risk of nephrotoxicity is very low with MRI contrast agents, even in patients with impaired renal function. However, these agents can induce nephrogenic systemic fibrosis (NSF), a rare but severe complication [282]. The risk of NSF depends upon the degree of impairment of the patient's renal function and the type of agent (stability of the Gadolinium bound to chelate).

Thus in patients with renal failure, it is recommended to consider an alternative imaging method that does not use iodinated or Gadolinium-containing contrast media. Since UCAs are not nephrotoxic (and no renal function assessment is required), CEUS should be considered in every case, especially if it offers the possibility of achieving enough diagnostic information, even with off-label CEUS uses. This is particularly the case in patients with severe renal failure, in whom CT or MRI should only be used in case of inconclusive examinations and strong clinical need.

\section{Technical Appendices}

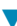

The number of systems capable of CEUS imaging has increased greatly in recent years and almost all manufacturers have im- plemented this technique in top level as well as, in many instances, in lower level machines. The technical details of the modalities which produce CEUS imaging are rapidly and continuously evolving so that any written list of information would not be complete. Accordingly, it is preferable that readers access such technical information directly from the websites of each manufacturer who supported this project. The links can be found on the EFSUMB webpage (http://www.efsumb.org/guide lines/ceus-manufacturers-links.pdf), but the content remains the responsibility of the respective manufacturers.

\section{Acknowledgements}

\section{$\nabla$}

The authors acknowledge the contribution of Dr. Ralph Faschinbauer who gave advice for the section on inflammatory joint disease.

The unrestricted financial support provided by the following companies (listed in alphabetical order) to support the preparation of this document is gratefully acknowledged:

B-K Medical, Bracco, Esaote, GE Healthcare, Medical Diagnostics, Hitachi, Lantheus Medical Imaging, Philips, Siemens, SuperSonic Imagine, Toshiba, Zonare.

\section{Affiliations}

1 Div. Internal Medicine, University of Bologna, Bologna Italy

2 US Section, Division of Surgery, Dept of Gastroenterology, Herlev Hospital, University of Copenhagen, Denmark

3 Div. Gastroenterology \& Oncology, Caritas Krankenhaus, Bad Mergentheim, Germany

${ }^{4}$ Imaging Sciences Department, Imperial College, Hammersmith Campus, London, UK

5 National Centre for Ultrasound in Gastroenterology, Department of Medicine, Haukeland University Hospital, Bergen, Norway and Institute of Medicine, University of Bergen, Norway

6 Radiology, Rigshospitalet, Copenhagen, Denmark

7 Radiology, Vivantes Klinikum Neukölln, Berlin, Germany

8 Radiology, General and University Hospital S.Orsola-Malpighi, Bologna, Italy Radiology, University of Trieste, Italy

10 Radiology, National Cancer Institute Fondazione G. Pascale, Naples, Italy

11 Radiology, Hôpital d'Enfants, Nancy, France

12 Radiology, Klinikum der Ludwig-Maximilians-Universität, München, Germany

13 Adult Radiology, Paris Descartes University \& Necker University Hospital, France

14 Radiology, University of Verona, Italy

15 Radiology, University “La Sapienza”, Rome, Italy

16 Div Neurology, St Josef Hospital, Bochum, Germany

17 Endoscopy Unit, Institut Paoli-Calmettes, Marseille, France

18 Div Gastroenterology, Klinikum Meiningen, Germany

19 Div. Gastroenterology \& Oncology, Caritas Krankenhaus, Bad Mergentheim, Germany

20 Radiology, University Hospital Regensburg, Germany

21 Radiology, Medical University Innsbruck, Austria

22 Imaging Department, Institut Gustave Roussy, Villejuif, France

23 Imaging Science Department, Imperial College London, UK

24 Internal Medicine, Rankweil, Austria

25 Gastroenterology, University of Medicine and Pharmacy, Craiova, Romania

26 Div. Neurology, Asklepios Klinik Nord, Heidberg, Germany

27 Radiology, King's College Hospital, London, UK

28 Joint Department of Physics, Royal Marsden Hospital, Sutton, UK

29 Div Gynaecology, University Hospitals Leuven, Belgium

30 Central Ultrasound Department, Krankenhaus Siloah, Hannover, Germany

\section{References}

1 Bertolotto M, Dalla Palma L, Quaia E et al. Characterization of unifocal liver lesions with pulse inversion harmonic imaging after Levovist injection: preliminary results. Eur Radiol 2000; 10: 1369-1376

2 Burns PN, Wilson SR, Simpson DH. Pulse inversion imaging of liver blood flow: improved method for characterizing focal masses with microbubble contrast. Invest Radiol 2000; 35: 58-71 
3 Albrecht T, Blomley M, Bolondi L et al. Guidelines for the use of contrast agents in ultrasound. January 2004. Ultraschall in Med 2004; 25 : 249-256

4 Claudon $M$, Cosgrove $D$, Albrecht $T$ et al. Guidelines and good clinical practice recommendations for contrast enhanced ultrasound (CEUS) - update 2008. Ultraschall in Med 2008; 29: 28-44

5 Nolsoe C, Piscaglia F, Dietrich CF et al. Reply to M. Piskunowicz, W. Kosiak and N. Irga's Letter to the Editor entitled "Primum non nocere? Why Can't We Use Second Generation Ultrasound Contrast Agents for the Examination of Children?". Ultraschall in Med 2011; 32: 83-86

6 Averkiou $M$, Powers J, Skyba $D$ et al. Ultrasound contrast imaging research. Ultrasound Q 2003; 19: 27-37

7 Schrope BA, Newhouse VL. Second harmonic ultrasonic blood perfusion measurement. Ultrasound Med Biol 1993; 19: 567-579

8 Simpson DH, Chin CT, Burns PN. Pulse inversion Doppler: a new method for detecting nonlinear echoes from microbubble contrast agents. IEEE Trans Ultrason Ferroelectr Freq Control 1999; 46: 372-382

9 Burns P, Powers J, Hope Simpson D et al. Harmonic Imaging: principals and preliminary results. Angiology 1996; 47: SA63-SA74

10 Dietrich $C F$, Ignee $A$, Hocke $M$ et al. Pitfalls and artefacts using contrast enhanced ultrasound. Z Gastroenterol 2011; 49: 350-356

11 Minimum training recommendations for the practice of medical ultrasound. Ultraschall in Med 2006; 27: 79-105

12 Minimum training requirements for the practice of Medical Ultrasound in Europe. Ultraschall in Med 2010; 31: 426-427

$13 \mathrm{Gilja} \mathrm{OH}$. Report Education and Professional Standard Commitee. In EFSUMB Newsletter. Ultraschall in Med 2009; 30: 86-87

14 Lassau N, Chami L, Benatsou B et al. Dynamic contrast-enhanced ultrasonography (DCE-US) with quantification of tumor perfusion: a new diagnostic tool to evaluate the early effects of antiangiogenic treatment. Eur Radiol 2007; 17 (Suppl 6): F89-F98

15 Piscaglia F, Bolondi L. The safety of Sonovue in abdominal applications: retrospective analysis of 23188 investigations. Ultrasound Med Biol 2006; 32: 1369-1375

16 ter Haar G. Safety and bio-effects of ultrasound contrast agents. Med Biol Eng Comput 2009; 47: 893-900

17 Blomley M, Claudon M, Cosgrove D. WFUMB Safety Symposium on Ultrasound Contrast Agents: clinical applications and safety concerns. Ultrasound Med Biol 2007; 33: 180-186

18 Barnett SB. Safe use of ultrasound contrast agents. Ultrasound Med Biol 2007; 33: 171-172

19 Main ML, Goldman JH, Grayburn PA. Ultrasound contrast agents: balancing safety versus efficacy. Expert Opin Drug Saf 2009; 8: 49-56

20 Main ML, Ryan AC, Davis TE et al. Acute mortality in hospitalized patients undergoing echocardiography with and without an ultrasound contrast agent (multicenter registry results in 4,300,966 consecutive patients). Am J Cardiol 2008; 102: 1742-1746

21 Dietrich CF, Braden B, Hocke $M$ et al. Improved characterisation of solitary solid pancreatic tumours using contrast enhanced transabdominal ultrasound. J Cancer Res Clin Oncol 2008; 134: 635-643

22 D’Onofrio M, Megibow AJ, Faccioli N et al. Comparison of contrast-enhanced sonography and MRI in displaying anatomic features of cystic pancreatic masses. Am J Roentgenol 2007; 189: 1435-1442

23 Kitano M, Kudo M, Maekawa K et al. Dynamic imaging of pancreatic diseases by contrast enhanced coded phase inversion harmonic ultrasonography. Gut 2004; 53: 854-859

24 D’Onofrio M, Malago R, Zamboni G et al. Contrast-enhanced ultrasonography better identifies pancreatic tumor vascularization than helical CT. Pancreatology 2005; 5: 398-402

25 Faccioli N, D'Onofrio M, Malago $R$ et al. Resectable pancreatic adenocarcinoma: depiction of tumoral margins at contrast-enhanced ultrasonography. Pancreas 2008; 37: 265-268

26 Grossjohann HS, Rappeport ED, Jensen C et al. Usefulness of contrastenhanced transabdominal ultrasound for tumor classification and tumor staging in the pancreatic head. Scand J Gastroenterol 2010; 45: 917-924

27 D’Onofrio M, Zamboni G, Tognolini A et al. Mass-forming pancreatitis: value of contrast-enhanced ultrasonography. World J Gastroenterol 2006; 12: 4181-4184

28 Kersting S, Konopke R, Kersting F et al. Quantitative perfusion analysis of transabdominal contrast-enhanced ultrasonography of pancreatic masses and carcinomas. Gastroenterology 2009; 137: 1903-1911

29 Numata K, Ozawa Y, Kobayashi $N$ et al. Contrast-enhanced sonography of pancreatic carcinoma: correlations with pathological findings. J Gastroenterol 2005; 40: 631-640
30 D'Onofrio $M$, Barbi E, Dietrich CF. Pancreatic multicenter ultrasound study (PAMUS). Eur J Radiol 2011 e-pub DOI: 10.1016/j. ejrad.2011.1001.1053

31 Tawada K, Yamaguchi T, Kobayashi A et al. Changes in tumor vascularity depicted by contrast-enhanced ultrasonography as a predictor of chemotherapeutic effect in patients with unresectable pancreatic cancer. Pancreas 2009; 38: 30-35

32 Kobayashi A, Yamaguchi T, Ishihara T et al. Evaluation of vascular signal in pancreatic ductal carcinoma using contrast enhanced ultrasonography: effect of systemic chemotherapy. Gut 2005; 54: 1047

33 D’Onofrio M, Mansueto G, Falconi M et al. Neuroendocrine pancreatic tumor: value of contrast enhanced ultrasonography. Abdom Imaging 2004; 29: 246-258

34 Malago R, D’Onofrio M, Zamboni GA et al. Contrast-enhanced sonography of nonfunctioning pancreatic neuroendocrine tumors. Am J Roentgenol 2009; 192: 424-430

35 Rickes S, Wermke W. Differentiation of cystic pancreatic neoplasms and pseudocysts by conventional and echo-enhanced ultrasound. J Gastroenterol Hepatol 2004; 19: 761-766

36 Itoh T, Hirooka Y, Itoh A et al. Usefulness of contrast-enhanced transabdominal ultrasonography in the diagnosis of intraductal papillary mucinous tumors of the pancreas. Am J Gastroenterol 2005; 100 : 144-152

37 Dietrich CF, Ignee A, Braden B et al. Improved differentiation of pancreatic tumors using contrast-enhanced endoscopic ultrasound. Clin Gastroenterol Hepatol 2008; 6: 590-597, e591

38 D'Onofrio M, Gallotti A, Pozzi Mucelli R. Imaging techniques in pancreatic tumors. Expert Rev Med Devices 2010; 7: 257-273

39 Ripolles T, Martinez MJ, Lopez E et al. Contrast-enhanced ultrasound in the staging of acute pancreatitis. Eur Radiol 2010; 20: 2518-2523

40 Becker D, Strobel D, Bernatik T et al. Echo-enhanced color- and powerDoppler EUS for the discrimination between focal pancreatitis and pancreatic carcinoma. Gastrointest Endosc 2001; 53: 784-789

41 Bhutani MS, Hoffman BJ, van Velse A et al. Contrast-enhanced endoscopic ultrasonography with galactose microparticles: SHU508 A (Levovist). Endoscopy 1997; 29: 635-639

42 Dietrich CF, Ignee A, Frey $H$. Contrast-enhanced endoscopic ultrasound with low mechanical index: a new technique. Z Gastroenterol 2005; 43: 1219-1223

43 Dietrich CF. Contrast-enhanced low mechanical index endoscopic ultrasound (CELMI-EUS). Endoscopy 2009; 41 (Suppl 2): E43-E44

44 Kitano M, Sakamoto H, Matsui U et al. A novel perfusion imaging technique of the pancreas: contrast-enhanced harmonic EUS (with video). Gastrointest Endosc 2008; 67: 141-150

45 Hocke M, Schmidt C, Zimmer B et al. [Contrast enhanced endosonography for improving differential diagnosis between chronic pancreatitis and pancreatic cancer]. Dtsch Med Wochenschr 2008; 133: 18881892

46 Hocke M, Schulze E, Gottschalk P et al. Contrast-enhanced endoscopic ultrasound in discrimination between focal pancreatitis and pancreatic cancer. World J Gastroenterol 2006; 12: 246-250

47 Kitano M, Takagi T, Sakamoto $H$. Dynamic imaging of pancreatic tumors by contrast-enhanced harmonic EUS with long-lasting contrast. Gastrointestinal Endoscopy 2009; 67: 141-150

48 Sakamoto H, Kitano M, Kamata Ket al. Diagnosis of pancreatic tumors by endoscopic ultrasonography. World J Radiol 2010; 2: 122-134

49 Hirooka $Y$, Itoh A, Kawashima $\mathrm{H}$ et al. Diagnosis of pancreatic disorders using contrast-enhanced endoscopic ultrasonography and endoscopic elastography. Clin Gastroenterol Hepatol 2009; 7: S63-S67

50 Saftoiu A, Iordache SA, Gheonea DI et al. Combined contrast-enhanced power Doppler and real-time sonoelastography performed during EUS, used in the differential diagnosis of focal pancreatic masses (with videos). Gastrointest Endosc 2010; 72: 739-747

51 Giovannini M. Contrast-enhanced endoscopic ultrasound and elastosonoendoscopy. Best Pract Res Clin Gastroenterol 2009; 23: 767-779

52 Hocke $M$, Ignee A, Topalidis $T$ et al. Contrast-enhanced endosonographic Doppler spectrum analysis is helpful in discrimination between focal chronic pancreatitis and pancreatic cancer. Pancreas 2007; 35: 286-288

53 Napoleon B, Alvarez-Sanchez MV, Gincoul R et al. Contrast-enhanced harmonic endoscopic ultrasound in solid lesions of the pancreas: results of a pilot study. Endoscopy 2010; 42: 564-570

54 Seicean A, Badea R, Stan-Iuga R et al. The added value of real-time harmonics contrast-enhanced endoscopic ultrasonography for the char- 
acterisation of pancreatic diseases in routine practice. J Gastrointestin Liver Dis 2010; 19: 99-104

55 Seicean A, Badea R, Stan-Iuga R et al. Quantitative contrast-enhanced harmonic endoscopic ultrasonography for the discrimination of solid pancreatic masses. Ultraschall in Med 2010; 31: 571-576

56 Fusaroli P, Spada A, Mancino MG et al. Contrast harmonic echo-endoscopic ultrasound improves accuracy in diagnosis of solid pancreatic masses. Clin Gastroenterol Hepatol 2010; 8: 629-634, e621 - e622

57 Beyer-Enke SA, Hocke M, Ignee A et al. Contrast enhanced transabdominal ultrasound in the characterisation of pancreatic lesions with cystic appearance. JOP 2010; 11: 427-433

58 Hocke M, Ignee A, Dietrich CF. Contrast-enhanced endoscopic ultrasound in the diagnosis of autoimmune pancreatitis. Endoscopy 2011; 43: 163-165

59 Plikat K, Klebl F, Buchner C et al. [Evaluation of intestinal hyperaemia in inflamed bowel by high resolution Contrast Harmonic Imaging (CHI)]. Ultraschall in Med 2004; 25: 257-262

60 Girlich C, Jung EM, Iesalnieks I et al. Quantitative assessment of bowel wall vascularisation in Crohn's disease with contrast-enhanced ultrasound and perfusion analysis. Clin Hemorheol Microcirc 2009; 43: 141-148

61 Schlottmann K, Kratzer W, Scholmerich J. Doppler ultrasound and intravenous contrast agents in gastrointestinal tract disorders: current role and future implications. Eur J Gastroenterol Hepatol 2005; 17: 263-275

62 Schreyer AG, Finkenzeller T, Gossmann $H$ et al. Microcirculation and perfusion with contrast enhanced ultrasound (CEUS) in Crohn's disease: first results with linear contrast harmonic imaging (CHI). Clin Hemorheol Microcirc 2008; 40: 143-155

63 Pauls S, Gabelmann A, Schmidt SA et al. Evaluating bowel wall vascularity in Crohn's disease: a comparison of dynamic MRI and wideband harmonic imaging contrast-enhanced low MI ultrasound. Eur Radiol 2006; 16: 2410-2417

64 Haber HP, Busch A, Ziebach R et al. Bowel wall thickness measured by ultrasound as a marker of Crohn's disease activity in children. Lancet 2000; 355: 1239-1240

65 Maconi G, Parente F, Bollani S et al. Abdominal ultrasound in the assessment of extent and activity of Crohn's disease: clinical significance and implication of bowel wall thickening. Am J Gastroenterol 1996; 91: 1604-1609

66 Spalinger J, Patriquin H, Miron MC et al. Doppler US in patients with crohn disease: vessel density in the diseased bowel reflects disease activity. Radiology 2000; 217: 787-791

67 Robotti D, Cammarota T, Debani P et al. Activity of Crohn disease: value of Color-Power-Doppler and contrast-enhanced ultrasonography. Abdom Imaging 2004; 29: 648-652

68 De Pascale A, Garofalo G, Perna $M$ et al. Contrast-enhanced ultrasonography in Crohn's disease. Radiol Med 2006; 111: 539-550

69 Serra C, Menozzi G, Labate AM et al. Ultrasound assessment of vascularization of the thickened terminal ileum wall in Crohn's disease patients using a low-mechanical index real-time scanning technique with a second generation ultrasound contrast agent. Eur J Radiol 2007; 62: 114-121

70 Migaleddu V, Scanu AM, Quaia E et al. Contrast-enhanced ultrasonographic evaluation of inflammatory activity in Crohn's disease. Gastroenterology 2009; 137: 43-52

71 Girlich C, Schacherer D, Jung EM et al. Comparison between a clinical activity index (Harvey-Bradshaw-Index), laboratory inflammation markers and quantitative assessment of bowel wall vascularization by contrast-enhanced ultrasound in Crohn's disease. Eur J Radiol 2011; epub DOI: 10.1016/j.ejrad.2011.02.054

72 Ripolles T, Martinez MJ, Paredes JM et al. Crohn disease: correlation of findings at contrast-enhanced US with severity at endoscopy. Radiology 2009; 253: 241-248

73 Guidi L, De Franco A, De Vitis I et al. Contrast-enhanced ultrasonography with SonoVue after infliximab therapy in Crohn's disease. Eur Rev Med Pharmacol Sci 2006; 10: 23-26

74 Kratzer W, von Tirpitz C, Mason $R$ et al. Contrast-enhanced power Doppler sonography of the intestinal wall in the differentiation of hypervascularized and hypovascularized intestinal obstructions in patients with Crohn's disease. J Ultrasound Med 2002; 21: 149-157, quiz 158 - 149

75 Wilson SR, Burns PN. Microbubble-enhanced US in body imaging: what role? Radiology 2010; 257: 24-39
76 Girlich C, Schacherer D, Lamby P et al. Innovations in contrast enhanced high resolution ultrasound improve sonographic imaging of the intestine. Clin Hemorheol Microcirc 2010; 45: 207-215

77 Henrich W, Meckies J, Friedmann W. Demonstration of a recto-vaginal fistula with the ultrasound contrast medium Echovist. Ultrasound Obstet Gynecol 2000; 15: 148-149

78 Dietrich $C F$. Significance of abdominal ultrasound in inflammatory bowel disease. Dig Dis 2009; 27: 482-493

79 Gorg C, Bert T. Contrast enhanced sonography of focal splenic lesions with a second-generation contrast agent. Ultraschall in Med 2005; 26: 470-477

80 Cavalli G, Re G, Casali AM. Red pulp arterial terminals in congestive splenomegaly. A morphometric study. Pathol Res Pract 1984; 178: 590-594

81 Lim AK, Patel N, Eckersley RJ et al. Evidence for spleen-specific uptake of a microbubble contrast agent: a quantitative study in healthy volunteers. Radiology 2004; 231: 785-788

82 Bertolotto M, Quaia E, Zappetti R et al. Differential diagnosis between splenic nodules and peritoneal metastases with contrast-enhanced ultrasound based on signal-intensity characteristics during the late phase. Radiol Med 2009; 114: 42-51

83 Gorg C, Bert T. Second-generation sonographic contrast agent for differential diagnosis of perisplenic lesions. Am J Roentgenol 2006; 186: 621-626

84 Gorg C, Graef C, Bert T. Contrast-enhanced sonography for differential diagnosis of an inhomogeneous spleen of unknown cause in patients with pain in the left upper quadrant. J Ultrasound Med 2006; 25 : 729-734

85 Liu GJ, Lu MD, Xie XY et al. Real-time contrast-enhanced ultrasound imaging of infected focal liver lesions. J Ultrasound Med 2008; 27 : 657-666

86 Chiavaroli R, Grima P, Tundo P. Characterization of nontraumatic focal splenic lesions using contrast-enhanced sonography. J Clin Ultrasound 2011; 39: 310-315

87 Catalano O, Sandomenico F, Matarazzo I et al. Contrast-enhanced sonography of the spleen. Am J Roentgenol 2005; 184: 1150-1156

88 Gorg C, Faoro C, Bert T et al. Contrast enhanced ultrasound of splenic lymphoma involvement. Eur J Radiol 2009; epub DOI: 10.1016/j. ejrad.2009.11.012

89 Sutherland T, Temple F, Hennessy $O$ et al. Contrast-enhanced ultrasound features of primary splenic lymphoma. J Clin Ultrasound 2010; 38: 317-319

$90 \mathrm{Yu} X, Y u$ J, Liang $P$ et al. Real-time contrast-enhanced ultrasound in diagnosing of focal spleen lesions. Eur J Radiol 2011; epub DOI: 10.1016/ j.ejrad.2010.12.052

91 von Herbay A, Barreiros AP, Ignee A et al. Contrast-enhanced ultrasonography with SonoVue: differentiation between benign and malignant lesions of the spleen. J Ultrasound Med 2009; 28: 421-434

92 Stang A, Keles H, Hentschke S et al. Differentiation of benign from malignant focal splenic lesions using sulfur hexafluoride-filled microbubble contrast-enhanced pulse-inversion sonography. Am J Roentgenol 2009; 193: 709-721

93 Tafuto S, Catalano O, Barba G et al. Real-time contrast-enhanced specific ultrasound in staging and follow-up of splenic lymphomas. Front Biosci 2006; 11: 2224-2229

94 Neesse A, Huth J, Kunsch S et al. Contrast-enhanced ultrasound pattern of splenic metastases - a retrospective study in 32 patients. Ultraschall in Med 2010; 31: 264-269

95 Picardi M, Soricelli A, Pane F et al. Contrast-enhanced harmonic compound US of the spleen to increase staging accuracy in patients with Hodgkin lymphoma: a prospective study. Radiology 2009; 251: 574582

96 Correas JM, Claudon M, Tranquart $F$ et al. The kidney: imaging with microbubble contrast agents. Ultrasound Q 2006; 22: 53-66

97 Bertolotto M, Martegani A, Aiani L et al. Value of contrast-enhanced ultrasonography for detecting renal infarcts proven by contrast enhanced CT. A feasibility study. Eur Radiol 2008; 18: 376-383

98 Bertolotto M, Catalano O. Contrast-enhanced Ultrasound: Past, Present, and Future. in: Advances in Ultrasound, An Issue of Ultrasound Clinics. Elsevier Science 2009; 4: 339-367

99 Blebea J, Zickler $R$, Volteas $N$ et al. Duplex imaging of the renal arteries with contrast enhancement. Vasc Endovascular Surg 2003; 37: 429436 
100 Tamai H, Takiguchi Y, Oka M et al. Contrast-enhanced ultrasonography in the diagnosis of solid renal tumors. J Ultrasound Med 2005; 24: $1635-1640$

101 Mazziotti S, Zimbaro F, Pandolfo A et al. Usefulness of contrast-enhanced ultrasonography in the diagnosis of renal pseudotumors. Abdom Imaging 2010; 35: 241-245

102 Ignee A, Straub B, Brix D et al. The value of contrast enhanced ultrasound (CEUS) in the characterisation of patients with renal masses. Clin Hemorheol Microcirc 2010; 46: 275-290

103 Quaia E, Bertolotto M, Cioffi V et al. Comparison of contrast-enhanced sonography with unenhanced sonography and contrast-enhanced CT in the diagnosis of malignancy in complex cystic renal masses. Am J Roentgenol 2008; 191: 1239-1249

104 Clevert DA, Minaifar N, Weckbach S et al. Multislice computed tomography versus contrast-enhanced ultrasound in evaluation of complex cystic renal masses using the Bosniak classification system. Clin Hemorheol Microcirc 2008; 39: 171-178

105 Park BK, Kim B, Kim SH et al. Assessment of cystic renal masses based on Bosniak classification: comparison of CT and contrast-enhanced US. Eur J Radiol 2007; 61: 310-314

106 Ascenti G, Mazziotti S, Zimbaro G et al. Complex cystic renal masses: characterization with contrast-enhanced US. Radiology 2007; 243: 158-165

107 Ignee A, Straub B, Schuessler G et al. Contrast enhanced ultrasound of renal masses. World J Radiol 2010; 2: 15-31

108 Wink MH, Lagerveld BW, Laguna MP et al. Cryotherapy for renal-cell cancer: diagnosis, treatment, and contrast-enhanced ultrasonography for follow-up. J Endourol 2006; 20: 456-458, discussion 458 459

109 Johnson DB, Duchene DA, Taylor GD et al. Contrast-enhanced ultrasound evaluation of radiofrequency ablation of the kidney: reliable imaging of the thermolesion. J Endourol 2005; 19: 248-252

110 Hoeffel C, Pousset M, Timsit MO et al. Radiofrequency ablation of renal tumours: diagnostic accuracy of contrast-enhanced ultrasound for early detection of residual tumour. Eur Radiol 2010; 20: 1812-1821

111 Meloni MF, Bertolotto M, Alberzoni C et al. Follow-up after percutaneous radiofrequency ablation of renal cell carcinoma: contrast-enhanced sonography versus contrast-enhanced CT or MRI. Am J Roentgenol 2008; 191: 1233-1238

112 Darge K. Voiding urosonography with ultrasound contrast agents for the diagnosis of vesicoureteric reflux in children. I. Procedure. Pediatr Radiol 2008; 38: 40-53

113 Darge K. Voiding urosonography with US contrast agents for the diagnosis of vesicoureteric reflux in children. II. Comparison with radiological examinations. Pediatr Radiol 2008; 38: 54-63, quiz 126 - 127

114 Darge $K$. Voiding urosonography with US contrast agent for the diagnosis of vesicoureteric reflux in children: an update. Pediatr Radiol 2010; 40: 956-962

115 Ascenti G, Zimbaro G, Mazziotti S et al. Harmonic US imaging of vesicoureteric reflux in children: usefulness of a second generation US contrast agent. Pediatr Radiol 2004; 34: 481-487

116 Lebowitz RL, Olbing H, Parkkulainen KV et al. International system of radiographic grading of vesicoureteric reflux. International Reflux Study in Children. Pediatr Radiol 1985; 15: 105-109

117 Duran C, Valera A, Alguersuari A et al. Voiding urosonography: the study of the urethra is no longer a limitation of the technique. Pediatr Radiol 2009; 39: 124-131

118 Moschouris H, Stamatiou K, Lampropoulou E et al. Imaging of the acute scrotum: is there a place for contrast-enhanced ultrasonography? Int Braz J Urol 2009; 35: 692-702, discussion 702 - 695

119 Bilagi P, Sriprasad S, Clarke JL et al. Clinical and ultrasound features of segmental testicular infarction: six-year experience from a single centre. Eur Radiol 2007; 17: 2810-2818

120 Sriprasad S, Kooiman GG, Muir GH et al. Acute segmental testicular infarction: differentiation from tumour using high frequency colour Doppler ultrasound. Br J Radiol 2001; 74: 965-967

121 Bertolotto M, Derchi LE, Sidhu PS et al. Acute segmental testicular infarction at contrast-enhanced ultrasound: early features and changes during follow-up. Am J Roentgenol 2011; 196: 834-841

122 Valentino $M$, Bertolotto $M$, Derchi $L$ et al. Role of contrast enhanced ultrasound in acute scrotal diseases. Eur Radiol 2011; epub DOI: $10.1007 / \mathrm{s} 00330-010-2039-5$

123 Bhatt S, Rubens DJ, Dogra VS. Sonography of benign intrascrotal lesions. Ultrasound Q 2006; 22: 121-136
124 Winter TC. There is a mass in the scrotum-what does it mean? Evaluation of the scrotal mass. Ultrasound Q 2009; 25: 195-205

125 Lock G, Schmidt C, Helmich F et al. Early Experience With Contrast-enhanced Ultrasound in the Diagnosis of Testicular Masses: A Feasibility Study. Urology 2011; 77: 1049-1053

126 Shah A, Lung PF, Clarke JL et al. Re: New ultrasound techniques for imaging of the indeterminate testicular lesion may avoid surgery completely. Clin Radiol 2010; 65: 496-497

127 Catalano O, Lobianco R, Sandomenico F et al. Splenic trauma: evaluation with contrast-specific sonography and a second-generation contrast medium: preliminary experience. J Ultrasound Med 2003; 22: 467-477

128 Valentino M, Serra C, Zironi G et al. Blunt abdominal trauma: emergency contrast-enhanced sonography for detection of solid organ injuries. Am J Roentgenol 2006; 186: 1361-1367

129 Catalano O, Aiani L, Barozzi L et al. CEUS in abdominal trauma: multicenter study. Abdom Imaging 2009; 34: 225-234

130 Reissig A, Gorg C, Mathis G. Transthoracic sonography in the diagnosis of pulmonary diseases: a systematic approach. Ultraschall in Med 2009; 30: 438-454, quiz 455 - 436

131 Gorg C. Transcutaneous contrast-enhanced sonography of pleuralbased pulmonary lesions. Eur J Radiol 2007; 64: 213-221

132 Bertolini FA, Goerg C, Mathis G. Echo contrast ultrasound in subpleural consolidations. Echo contrast ultrasound in subpleural consolidations 2008; 18 (Suppl S 395): Abstract

133 Gorg C, Bert T, Gorg K. Contrast-enhanced sonography for differential diagnosis of pleurisy and focal pleural lesions of unknown cause. Chest 2005; 128: 3894-3899

134 Gorg C, Bert T, Kring R et al. Transcutaneous contrast enhanced sonography of the chest for evaluation of pleural based pulmonary lesions: experience in 137 patients. Ultraschall in Med 2006; 27: 437 444

135 Clevert DA, Johnson T, Michaely H et al. High-grade stenoses of the internal carotid artery: comparison of high-resolution contrast enhanced 3D MRA, duplex sonography and power Doppler imaging. Eur J Radiol 2006; 60: 379-386

136 Jung EM, Kubale R, Ritter G et al. Diagnostics and characterisation of preocclusive stenoses and occlusions of the internal carotid artery with B-flow. Eur Radiol 2007; 17: 439-447

137 Clevert DA, Johnson T, Jung EM et al. Color Doppler, power Doppler and B-flow ultrasound in the assessment of ICA stenosis: Comparison with 64-MD-CT angiography. Eur Radiol 2007; 17: 2149-2159

138 Wechsler LR. Ulceration and carotid artery disease. Stroke 1988; 19: 650-653

139 Clevert DA, Horng A, Jung EM et al. Contrast-enhanced ultrasound versus conventional ultrasound and MS-CT in the diagnosis of abdominal aortic dissection. Clin Hemorheol Microcirc 2009; 43: 129-139

140 Clevert DA, Sommer WH, Zengel P et al. Imaging of carotid arterial diseases with contrast-enhanced ultrasound (CEUS). Eur J Radiol 2011; epub DOI: 10.1016/j.ejrad.2010.12.103

141 ten Kate GL, Sijbrands EJ, Staub D et al. Noninvasive imaging of the vulnerable atherosclerotic plaque. Curr Probl Cardiol 2010; 35: 556-591

142 Clevert DA, Sommer WH, Helck A et al. Duplex and contrast enhanced ultrasound (CEUS) in evaluation of in-stent restenosis after carotid stenting. Clin Hemorheol Microcirc 2011; in press

143 Massalha K, Goyen M, Rudofsky G. Stenosis-jet can cause a dissection of the superficial femoral artery. Vasa 1999; 28: 131-133

144 Koennecke HC, Trocio Jr. SH, Mast H et al. Microemboli on transcrania Doppler in patients with spontaneous carotid artery dissection. J Neuroimaging 1997; 7: 217-220

145 Clevert DA, Kubisch C, Meimarakis G et al. Improved visualization of carotid-jugular arteriovenous fistula by contrast-enhanced ultrasound. Ultraschall in Med 2010; 31: 610-612

146 Jaff MR, Goldmakher GV, Lev MH et al. Imaging of the carotid arteries: the role of duplex ultrasonography, magnetic resonance arteriography, and computerized tomographic arteriography. Vasc Med 2008; 13: $281-292$

147 Coli S, Magnoni M, Sangiorgi G et al. Contrast-enhanced ultrasound imaging of intraplaque neovascularization in carotid arteries: correlation with histology and plaque echogenicity. J Am Coll Cardiol 2008; 52: 223-230

148 Giannoni MF, Vicenzini E, Citone $M$ et al. Contrast carotid ultrasound for the detection of unstable plaques with neoangiogenesis: a pilot study. Eur J Vasc Endovasc Surg 2009; 37: 722-727 
149 Staub D, Schinkel AF, Coll B et al. Contrast-enhanced ultrasound imaging of the vasa vasorum: from early atherosclerosis to the identification of unstable plaques. JACC Cardiovasc Imaging 2010; 3: 761-771

150 Staub D, Patel MB, Tibrewala A et al. Vasa vasorum and plaque neovascularization on contrast-enhanced carotid ultrasound imaging correlates with cardiovascular disease and past cardiovascular events. Stroke 2010; 41: 41-47

151 Catalano O, Lobianco R, Cusati B et al. Contrast-enhanced sonography for diagnosis of ruptured abdominal aortic aneurysm. Am J Roentgenol 2005; 184: 423-427

152 Spittell PC, Spittell Jr. JA, Joyce JW et al. Clinical features and differential diagnosis of aortic dissection: experience with 236 cases (1980 through 1990). Mayo Clin Proc 1993; 68: 642-651

153 Clevert DA, Rupp N, Reiser M et al. Improved diagnosis of vascular dissection by ultrasound B-flow: a comparison with color-coded Doppler and power Doppler sonography. Eur Radiol 2005; 15: 342-347

154 Clevert DA, Stickel M, Johnson T et al. Imaging of aortic abnormalities with contrast-enhanced ultrasound. A pictorial comparison with CT. Eur Radiol 2007; 17: 2991-3000

155 Clevert DA, Stickel M, Kopp R et al. Contrast-enhanced ultrasound versus $C T$ and operative findings in evaluation of renal vein aneurysm with AV fistula. Clin Hemorheol Microcirc 2008; 39: 147-154

156 Arrive L, Correas JM, Leseche $G$ et al. Inflammatory aneurysms of the abdominal aorta: CT findings. Am J Roentgenol 1995; 165: 14811484

157 Pennell RC, Hollier LH, Lie JT et al. Inflammatory abdominal aortic aneurysms: a thirty-year review. J Vasc Surg 1985; 2: 859-869

158 Walker DI, Bloor K, Williams G et al. Inflammatory aneurysms of the abdominal aorta. Br J Surg 1972; 59: 609-614

159 Witz M, Korzets Z. Inflammatory abdominal aortic aneurysms. Isr Med Assoc J 2005; 7: 385-387

160 Crawford JL, Stowe CL, Safi HJ et al. Inflammatory aneurysms of the aorta. J Vasc Surg 1985; 2: 113-124

161 Clevert DA, Schick $K$, Chen MH et al. Role of contrast enhanced ultrasound in detection of abdominal aortic abnormalities in comparison with multislice computed tomography. Chin Med J (Engl) 2009; 122: 858-864

162 Thurnher S, Cejna M. Imaging of aortic stent-grafts and endoleaks. Radiol Clin North Am 2002: 40: 799-833

163 Clevert DA, Minaifar N, Weckbach S et al. Color duplex ultrasound and contrast-enhanced ultrasound in comparison to MS-CT in the detection of endoleak following endovascular aneurysm repair. Clin Hemorheol Microcirc 2008; 39: 121-132

164 d'Audiffret A, Desgranges $P$, Kobeiter DH et al. Follow-up evaluation of endoluminally treated abdominal aortic aneurysms with duplex ultrasonography: validation with computed tomography. J Vasc Surg 2001; 33: 42-50

165 Sommer WH, Hoffmann RT, Becker CR et al. Comparison of time-resolved CT-angiography, contrast-enhanced ultrasound and digital subtraction angiography in a patient with a small type II endoleak after endovascular aneurysm repair. Clin Hemorheol Microcirc 2010; 45: 19-25

166 McLafferty RB, McCrary BS, Mattos MA et al. The use of color-flow duplex scan for the detection of endoleaks. J Vasc Surg 2002; 36: 100104

167 Greenfield AL, Halpern EJ, Bonn J et al. Application of duplex US for characterization of endoleaks in abdominal aortic stent-grafts: report of five cases. Radiology 2002; 225: 845-851

168 Jung EM, Rennert J, Fellner C et al. Detection and characterization of endoleaks following endovascular treatment of abdominal aortic aneurysms using contrast harmonic imaging $(\mathrm{CHI})$ with quantitative perfusion analysis (TIC) compared to CT angiography (CTA). Ultraschall in Med 2010; 31: 564-570

169 Pfister K, Rennert J, Uller $W$ et al. Contrast harmonic imaging ultrasound and perfusion imaging for surveillance after endovascular abdominal aneurysm repair regarding detection and characterization of suspected endoleaks. Clin Hemorheol Microcirc 2009; 43: 119128

170 Ten Bosch JA, Rouwet EV, Peters CT et al. Contrast-enhanced ultrasound versus computed tomographic angiography for surveillance of endovascular abdominal aortic aneurysm repair. J Vasc Interv Radiol 2010: 21: 638-643

171 Seidel G, Meairs S. Ultrasound contrast agents in ischemic stroke. Cerebrovasc Dis 2009; 27 (Suppl 2): 25-39
172 Seidel G, Kaps M. Harmonic imaging of the vertebrobasilar system. Stroke 1997; 28: 1610-1613

173 Postert T, Braun B, Meves S et al. Contrast-enhanced transcranial color-coded sonography in acute hemispheric brain infarction. Stroke 1999; 30: 1819-1826

174 Droste DW, Jurgens R, Weber S et al. Benefit of echocontrast-enhanced transcranial color-coded duplex ultrasound in the assessment of intracranial collateral pathways. Stroke 2000; 31: 920-923

175 Seidel G, Cangur H, Meyer-Wiethe K et al. On the ability of ultrasound parametric perfusion imaging to predict the area of infarction in acute ischemic stroke. Ultraschall in Med 2006; 27: 543-548

176 Eyding J, Krogias C, Schollhammer $M$ et al. Contrast-enhanced ultrasonic parametric perfusion imaging detects dysfunctional tissue at risk in acute MCA stroke. J Cereb Blood Flow Metab 2006; 26: 576-582

177 Krogias C, Hennebohl C, Geier B et al. Transcranial ultrasound perfusion imaging and perfusion-MRI - a pilot study on the evaluation of cerebral perfusion in severe carotid artery stenosis. Ultrasound Med Biol 2010; 36: 1973-1980

178 Krogias C, Meves SH, Hansen C et al. Ultrasound Perfusion Imaging of the Brain-Routine and Novel Applications: Uncommon Cases and Review of the Literature. J Neuroimaging 2010; 21: 255-258

179 Eggers J, Konig IR, Koch B et al. Sonothrombolysis with transcranial color-coded sonography and recombinant tissue-type plasminogen activator in acute middle cerebral artery main stem occlusion: results from a randomized study. Stroke 2008; 39: 1470-1475

180 FitzGerald O, Bresnihan B. Synovial membrane cellularity and vascularity. Ann Rheum Dis 1995; 54: 511-515

181 Klauser A, Frauscher F, Schirmer M. Value of contrast-enhanced power Doppler ultrasonography (US) of the metacarpophalangeal joints on rheumatoid arthritis. Eur Radiol 2004; 14: 545-546, author reply $547-548$

182 Klauser AS, Peetrons P. Developments in musculoskeletal ultrasound and clinical applications. Skeletal Radiol 2010; 39: 1061-1071

183 De Zordo T, Mlekusch SP, Feuchtner GM et al. Value of contrast-enhanced ultrasound in rheumatoid arthritis. Eur J Radiol 2007; 64: 222-230

184 Taylor PC. The value of sensitive imaging modalities in rheumatoid arthritis. Arthritis Res Ther 2003; 5: 210-213

185 Hermann KG, Backhaus M, Schneider $U$ et al. Rheumatoid arthritis of the shoulder joint: comparison of conventional radiography, ultrasound, and dynamic contrast-enhanced magnetic resonance imaging. Arthritis Rheum 2003; 48: 3338-3349

186 Klauser A, Frauscher F, Schirmer $M$ et al. The value of contrast-enhanced color Doppler ultrasound in the detection of vascularization of finger joints in patients with rheumatoid arthritis. Arthritis Rheum 2002; 46: 647-653

187 Doria AS, Kiss MH, Lotito AP et al. Juvenile rheumatoid arthritis of the knee: evaluation with contrast-enhanced color Doppler ultrasound Pediatr Radiol 2001; 31: 524-531

188 Magarelli N, Guglielmi G, Di Matteo L et al. Diagnostic utility of an echo-contrast agent in patients with synovitis using power Doppler ultrasound: a preliminary study with comparison to contrast-enhanced MRI. Eur Radiol 2001; 11: 1039-1046

189 Klauser A, Halpern EJ, Frauscher F et al. Inflammatory low back pain: high negative predictive value of contrast-enhanced color Doppler ultrasound in the detection of inflamed sacroiliac joints. Arthritis Rheum 2005; 53: 440-444

190 Ostergaard $M$, Hansen $M$, Stoltenberg $M$ et al. Magnetic resonance imaging-determined synovial membrane volume as a marker of disease activity and a predictor of progressive joint destruction in the wrists of patients with rheumatoid arthritis. Arthritis Rheum 1999; 42: 918-929

191 Klauser A, Demharter J, De Marchi A et al. Contrast enhanced grayscale sonography in assessment of joint vascularity in rheumatoid arthritis: results from the IACUS study group. Eur Radiol 2005; 15: 2404-2410

192 Schueller-Weidekamm C, Krestan C, Schueller G et al. Power Doppler sonography and pulse-inversion harmonic imaging in evaluation of rheumatoid arthritis synovitis. Am J Roentgenol 2007; 188: 504-508

193 Klauser AS, Franz M, Arora R et al. Detection of vascularity in wrist tenosynovitis: power doppler ultrasound compared with contrast-enhanced grey-scale ultrasound. Arthritis Res Ther 2010; 12: R209

194 Wamser G, BohndorfK, Vollert K et al. Power Doppler sonography with and without echo-enhancing contrast agent and contrast-enhanced MRI for the evaluation of rheumatoid arthritis of the shoulder joint: 
differentiation between synovitis and joint effusion. Skeletal Radiol 2003; 32: 351-359

195 Carotti M, Salaffi F, Manganelli P et al. Power Doppler sonography in the assessment of synovial tissue of the knee joint in rheumatoid arthritis: a preliminary experience. Ann Rheum Dis 2002; 61: 877-882

196 Hau M, Kneitz C, Tony HP et al. High resolution ultrasound detects a decrease in pannus vascularisation of small finger joints in patients with rheumatoid arthritis receiving treatment with soluble tumour necrosis factor alpha receptor (etanercept). Ann Rheum Dis 2002; 61: $55-58$

197 Song IH, Althoff CE, Hermann KG et al. Contrast-enhanced ultrasound in monitoring the efficacy of a bradykinin receptor 2 antagonist in painful knee osteoarthritis compared with MRI. Ann Rheum Dis 2009; 68: 75-83

198 Qvistgaard E, Kristoffersen H, Terslev L et al. Guidance by ultrasound of intra-articular injections in the knee and hip joints. Osteoarthritis Cartilage 2001; 9: 512-517

199 Qvistgaard E, Rogind H, Torp-Pedersen S et al. Quantitative ultrasonography in rheumatoid arthritis: evaluation of inflammation by Doppler technique. Ann Rheum Dis 2001; 60: 690-693

200 Lanzani C, Savasi V, Leone FP et al. Two-dimensional HyCoSy with contrast tuned imaging technology and a second-generation contrast media for the assessment of tubal patency in an infertility program. Fertil Steril 2009; 92: 1158-1161

201 Boudghene FP, Bazot M, Robert Y et al. Assessment of Fallopian tube patency by HyCoSy: comparison of a positive contrast agent with saline solution. Ultrasound Obstet Gynecol 2001; 18: 525-530

202 Tamasi F, Weidner A, Domokos $N$ et al. ECHOVIST-200 enhanced hystero-sonography: a new technique in the assessment of infertility. Eur J Obstet Gynecol Reprod Biol 2005; 121: 186-190

203 Foschi FG, Piscaglia F, Pompili M et al. Real-time contrast-enhanced ultrasound-a new simple tool for detection of peritoneal-pleural communications in hepatic hydrothorax. Ultraschall in Med 2008; 29 : 538-542

204 Tamano M, Hashimoto T, Kojima K et al. Diagnosis of hepatic hydrothorax using contrast-enhanced ultrasonography with intraperitoneal injection of Sonazoid. J Gastroenterol Hepatol 2010; 25: 383386

205 Ignee A, Baum U, Schuessler G et al. Contrast-enhanced ultrasound-guided percutaneous cholangiography and cholangiodrainage (CEUSPTCD). Endoscopy 2009; 41: 725-726

$206 \mathrm{Mao}$, Xu EJ, Li K et al. Usefulness of contrast-enhanced ultrasound in the diagnosis of biliary leakage following T-tube removal. J Clin Ultrasound 2009; 38: 38-40

207 Zuber-Jerger I, Endlicher E, Scholmerich J et al. Endoscopic retrograde cholangiography with contrast ultrasonography. Endoscopy 2008; 40 (Suppl 2): E202

208 Zengel P, Berghaus A, Weiler C et al. Intraductally applied contrast-enhanced ultrasound (IA-CEUS) for evaluating obstructive disease and secretory dysfunction of the salivary glands. Eur Radiol 2011; 21: 1339-1348

209 Volkmer BG, Nesslauer T, Kufer R et al. [Diagnosis of vesico-intestinal fistulas by contrast medium enhanced 3-D ultrasound]. Ultraschall in Med 2001; 22: 81-86

210 Chew SS, Yang JL, Newstead GL et al. Anal fistula: Levovist-enhanced endoanal ultrasound: a pilot study. Dis Colon Rectum 2003; 46: 377-384

211 Moritz JD, Ludwig A, Oestmann JW. Contrast-enhanced color Doppler sonography for evaluation of enlarged cervical lymph nodes in head and neck tumors. Am J Roentgenol 2000; 174: 1279-1284

212 Benzel W, Zenk J, Winter M et al. [Color Doppler ultrasound studies of benign and malignant lymph nodes]. HNO 1996; 44: 666-671

213 Schmid-Wendtner MH, Partscht K, Korting HC et al. Improved differentiation of benign and malignant lymphadenopathy in patients with cutaneous melanoma by contrast-enhanced color Doppler sonography. Arch Dermatol 2002; 138: 491-497

214 Schulte-Altedorneburg G, Demharter J, Linne R et al. Does ultrasound contrast agent improve the diagnostic value of colour and power Doppler sonography in superficial lymph node enlargement? Eur J Radiol 2003; 48: 252-257

215 Schmid-Wendtner MH, Dill-Muller D, Baumert J et al. Lymph node metastases in patients with cutaneous melanoma: improvements in diagnosis by signal-enhanced color Doppler sonography. Melanoma Res 2004; 14: 269-276
216 Zenk J, Bozzato A, Steinhart H et al. Metastatic and inflammatory cervical lymph nodes as analyzed by contrast-enhanced color-coded Doppler ultrasonography: quantitative dynamic perfusion patterns and histopathologic correlation. Ann Otol Rhinol Laryngol 2005; 114: 43-47

217 Stoeckelhuber BM, Wiesmann M, Berg SA et al. [Sonography of enlarged lymph nodes: Pathogenetic categorization using contrast enhanced power Doppler sonography]. Hautarzt 2006; 57: 610-617

218 Weskott HP. Emerging roles for contrast-enhanced ultrasound. Clin Hemorheol Microcirc 2008; 40: 51-71

219 Steppan I, Reimer D, Muller-Holzner E et al. Breast cancer in women: evaluation of benign and malignant axillary lymph nodes with contrast-enhanced ultrasound. Ultraschall in Med 2010; 31: 63-67

220 Ouyang $\mathrm{Q}$ Chen $\mathrm{L}$, Zhao $\mathrm{H}$ et al. Detecting metastasis of lymph nodes and predicting aggressiveness in patients with breast carcinomas. J Ultrasound Med 2010; 29: 343-352

221 Stramare R, Scagliori E, Mannucci $M$ et al. The role of contrast-enhanced gray-scale ultrasonography in the differential diagnosis of superficial lymph nodes. Ultrasound Q 2010; 26: 45-51

222 Yu M, Liu Q Song HP et al. Clinical application of contrast-enhanced ultrasonography in diagnosis of superficial lymphadenopathy. J Ultrasound Med 2010; 29: 735-740

223 Rubaltelli L, Beltrame V, Tregnaghi A et al. Contrast-enhanced ultrasound for characterizing lymph nodes with focal cortical thickening in patients with cutaneous melanoma. Am J Roentgenol 2011; 196 : W8-W12

224 Hocke $M$, Menges $M$, Topalidis $T$ et al. Contrast-enhanced endoscopic ultrasound in discrimination between benign and malignant mediastinal and abdominal lymph nodes. J Cancer Res Clin Oncol 2008; 134: 473-480

225 Nakase K, Yamamoto K, Hiasa A et al. Contrast-enhanced ultrasound examination of lymph nodes in different types of lymphoma. Cancer Detect Prev 2006; 30: 188-191

226 Rue Nielsen K, Klyver H, Hougaard Chakera A et al. Sentinel node detection in melanomas using contrast-enhanced ultrasound. Acta Radiol 2009; 50: 412-417

227 Wang Y, Wang W, Li J et al. Gray-scale contrast-enhanced ultrasonography of sentinel lymph nodes in a metastatic breast cancer model. Acad Radiol 2009; 16: 957-962

228 Omoto K, Matsunaga $\mathrm{H}$, Take $\mathrm{N}$ et al. Sentinel node detection method using contrast-enhanced ultrasonography with sonazoid in breast cancer: preliminary clinical study. Ultrasound Med Biol 2009; 35: 1249-1256

229 Sever A, Jones S, Cox $K$ et al. Preoperative localization of sentinel lymph nodes using intradermal microbubbles and contrast-enhanced ultrasonography in patients with breast cancer. Br J Surg 2009; 96: 1295-1299

230 Nielsen KR. Studies on radionuclide imaging and contrast ultrasound for sentinel node diagnostics in breast cancer and melanoma. Dan Med Bull 2011; 58: B4225

231 Sever AR, Mills $P$, Jones SE et al. Preoperative sentinel node identification with ultrasound using microbubbles in patients with breast cancer. Am J Roentgenol 2010; 196: 251-256

232 Tozer GM. Measuring tumour vascular response to antivascular and antiangiogenic drugs. Br J Radiol 2003; 76 Spec No 1: S23-S35

233 Eisenhauer EA, Therasse P, Bogaerts J et al. New response evaluation criteria in solid tumours: revised RECIST guideline (version 1.1). Eur J Cancer 2009; 45: 228-247

234 Zhu AX, Holalkere NS, Muzikansky A et al. Early antiangiogenic activity of bevacizumab evaluated by computed tomography perfusion scan in patients with advanced hepatocellular carcinoma. Oncologist 2008; 13: 120-125

235 Peronneau P, Lassau N, Leguerney I et al. Contrast ultrasonography: necessity of linear data processing for the quantification of tumor vascularization. Ultraschall in Med 2010; 31: 370-378

236 Averkiou M, Lampaskis M, Kyriakopoulou K et al. Quantification of tumor microvascularity with respiratory gated contrast enhanced ultrasound for monitoring therapy. Ultrasound Med Biol 2010; 36: 68-77

237 Williams R, Hudson JM, Lloyd BA et al. Dynamic Microbubble Contrastenhanced US to Measure Tumor Response to Targeted Therapy: A Proposed Clinical Protocol with Results from Renal Cell Carcinoma Patients Receiving Antiangiogenic Therapy. Radiology 2011; 260: 581590 
238 Lassau N, Lamuraglia M, Vanel D et al. Doppler US with perfusion software and contrast medium injection in the early evaluation of isolated limb perfusion of limb sarcomas: prospective study of 49 cases. Ann Oncol 2005; 16: 1054-1060

239 Cosgrove D, Lassau N. Imaging of perfusion using ultrasound. Eur J Nucl Med Mol Imaging 2010; 37 (Suppl 1): S65-S85

240 Lamuraglia M, Escudier B, Chami L et al. To predict progression-free survival and overall survival in metastatic renal cancer treated with sorafenib: pilot study using dynamic contrast-enhanced Doppler ultrasound. Eur J Cancer 2006; 42: 2472-2479

241 Lassau N, Lamuraglia M, Chami L et al. Gastrointestinal stromal tumors treated with imatinib: monitoring response with contrast-enhanced sonography. Am J Roentgenol 2006; 187: 1267-1273

242 De Giorgi U, Aliberti C, Benea $G$ et al. Effect of angiosonography to monitor response during imatinib treatment in patients with metastatic gastrointestinal stromal tumors. Clin Cancer Res 2005; 11: 6171-6176

243 Escudier B, Lassau N, Angevin E et al. Phase I trial of sorafenib in combination with IFN alpha-2a in patients with unresectable and/or metastatic renal cell carcinoma or malignant melanoma. Clin Cancer Res 2007; 13: 1801-1809

244 Lassau N, Koscielny S, Albiges L et al. Metastatic renal cell carcinoma treated with sunitinib: early evaluation of treatment response using dynamic contrast-enhanced ultrasonography. Clin Cancer Res 2010; 16: $1216-1225$

245 Lassau N, Chami L, Koscielny S et al. Quantitative functional imaging by Dynamic Contrast Enhanced Ultrasonography (DCE-US) in GIST patients treated with masatinib. Invest New Drugs 2010; epub DOI: 10.1007/s10637-010-9592-2

246 Lassau N, Koscielny S, Chami L et al. Advanced hepatocellular carcinoma: early evaluation of response to bevacizumab therapy at dynamic contrast-enhanced US with quantification - preliminary results. Radiology 2011; 258: 291-300

247 Lassau N, Chami L, Chebil M et al. Dynamic contrast-enhanced ultrasonography (DCE-US) and anti-angiogenic treatments. Discov Med 2011; 11: 18-24

248 Casali PG, Blay JY Experts ECECPo. Gastrointestinal stromal tumours: ESMO clinical practice guidelines for diagnosis, treatment and follow-up. Ann Oncol 2010; (Suppl 5): v98-v102

249 Cosgrove DO, Bamber JC, Davey JB et al. Color Doppler signals from breast tumors. Work in progress. Radiology 1990; 176: 175-180

250 Sorelli PG, Cosgrove DO, Svensson WE et al. Can contrast-enhanced sonography distinguish benign from malignant breast masses? J Clin Ultrasound 2010; 38: 177-181

251 Balleyguier C, Opolon P, Mathieu MC et al. New potential and applications of contrast-enhanced ultrasound of the breast: Own investigations and review of the literature. Eur J Radiol 2009; 69: 14-23

252 Liu H, Jiang YX, Liu JB et al. Evaluation of breast lesions with contrastenhanced ultrasound using the microvascular imaging technique: initial observations. Breast 2008; 17: 532-539

253 Caproni N, Marchisio F, Pecchi A et al. Contrast-enhanced ultrasound in the characterisation of breast masses: utility of quantitative analysis in comparison with MRI. Eur Radiol 2009; 20: 1384-1395

254 Friedrich-Rust M, Glasemann T, Polta A et al. Differentiation between Benign and Malignant Adrenal Mass using Contrast-Enhanced Ultrasound. Ultraschall in Med 2011; epub DOI: 10.1055/s-0031-1273408

255 Dietrich CF, Ignee A, Barreiros AP et al. Contrast-enhanced ultrasound for imaging of adrenal masses. Ultraschall in Med 2010; 31: 163-168

256 Testa AC, Ferrandina G, Fruscella $E$ et al. The use of contrasted transvaginal sonography in the diagnosis of gynecologic diseases: a preliminary study. J Ultrasound Med 2005; 24: 1267-1278

257 Lieng M, Qvigstad E, Dahl GF et al. Flow differences between endometrial polyps and cancer: a prospective study using intravenous contrast-enhanced transvaginal color flow Doppler and three-dimensional power Doppler ultrasound. Ultrasound Obstet Gynecol 2008; 32: 935-940

258 Marret H, Sauget S, Giraudeau B et al. Contrast-enhanced sonography helps in discrimination of benign from malignant adnexal masses. J Ultrasound Med 2004; 23: 1629-1639, quiz 1641 - 1642

259 Orden MR, Jurvelin JS, Kirkinen PP. Kinetics of a US contrast agent in benign and malignant adnexal tumors. Radiology 2003; 226: 405410

260 Testa AC, Timmerman D, Exacoustos C et al. The role of CnTI-SonoVue in the diagnosis of ovarian masses with papillary projections: a preliminary study. Ultrasound Obstet Gynecol 2007; 29: 512-516
261 Caruso G, Salvaggio G, Campisi A et al. Bladder tumor staging: comparison of contrast-enhanced and gray-scale ultrasound. Am J Roentgenol 2010; 194: 151-156

262 Drudi FM, Cantisani V, Liberatore M et al. Role of low-mechanical index CEUS in the differentiation between low and high grade bladder carcinoma: a pilot study. Ultraschall in Med 2010; 31: 589-595

263 Nicolau C, Bunesch L, Sebastia C et al. Diagnosis of bladder cancer: contrast-enhanced ultrasound. Abdom Imaging 2010; 35: 494-503

264 Lefevre F, Correas JM, Briancon S et al. Contrast-enhanced sonography of the renal transplant using triggered pulse-inversion imaging: preliminary results. Ultrasound Med Biol 2002; 28: 303-314

265 Mitterberger MJ, Aigner F, Horninger W et al. Comparative efficiency of contrast-enhanced colour Doppler ultrasound targeted versus systematic biopsy for prostate cancer detection. Eur Radiol 2010; 20: 2791-2796

266 Wink M, Frauscher F, Cosgrove D et al. Contrast-enhanced ultrasound and prostate cancer, a multicentre European research coordination project. Eur Urol 2008; 54: 982-992

267 Aigner F, Pallwein L, Mitterberger $M$ et al. Contrast-enhanced ultrasonography using cadence-contrast pulse sequencing technology for targeted biopsy of the prostate. BJU Int 2009; 103: 458-463

268 Sano F, Terao H, Kawahara $T$ et al. Contrast-enhanced ultrasonography of the prostate: various imaging findings that indicate prostate cancer. BJU Int 2010; 107: 1404-1410

269 Strazdina A, Krumina G, Sperga M. The value and limitations of contrast-enhanced ultrasound in detection of prostate cancer. Anticancer Res 2011; 31: 1421-1426

270 Davis PM, Gloviczki P, Cherry Jr KJ et al. Aorto-caval and ilio-iliac arteriovenous fistulae. Am J Surg 1998; 176: 115-118

271 Gardiner MD, NanchahalJ. Strategies to ensure success of microvascular free tissue transfer. J Plast Reconstr Aesthet Surg 2010; 63: e665673

272 Prantl L, Pfister K, Kubale R et al. Value of high resolution ultrasound and contrast enhanced US pulse inversion imaging for the evaluation of the vascular integrity of free-flap grafts. Clin Hemorheol Microcirc 2007; 36: 203-216

273 Prantl L, Schmitt S, Geis S et al. Contrast harmonic ultrasound and indocyanine-green fluorescence video angiography for evaluation of dermal and subdermal microcirculation in free parascapular flaps. Clin Hemorheol Microcirc 2008; 38: 105-118

274 Prantl L, Schreml S, Walter M et al. Evaluation of microcirculation of free flaps of the lower leg by contrast harmonic imaging (CHI) with time intensity curve (TIC) analysis. Clin Hemorheol Microcirc 2008; 39: $343-350$

275 Lamby P, Prantl L, Schreml S et al. Improvements in high resolution ultrasound for postoperative investigation of capillary microperfusion after free tissue transfer. Clin Hemorheol Microcirc 2009; 43: 35-49

276 Prantl L, Fellner C, Jung ME. Evaluation of free flap perfusion with dynamic contrast-enhanced magnetic resonance imaging. Plast Reconstr Surg 2010; 126: 100e-101e

277 Jung EM, Prantl L, Schreyer AG et al. New perfusion imaging of tissue transplants with Contrast Harmonic Ultrasound Imaging (CHI) and Magnetic Resonance Imaging (MRI) in comparison with laser-induced Indocyanine Green (ICG) fluorescence angiography. Clin Hemorheol Microcirc 2009; 43: 19-33

278 Geis S, Prantl L, Gehmert S et al. TTP (time to PEAK) and RBV (regional blood volume) as valuable parameters to detect early flap failure. Clin Hemorheol Microcirc 2011; 47: 1-14

279 Fellner C, Jung EM, Prantl L. Dynamic contrast-enhanced MRI as a valuable non-invasive tool to evaluate tissue perfusion of free flaps: Preliminary results. Clin Hemorheol Microcirc 2010; 46: 77-87

280 Lamby P, Prantl L, Fellner C et al. Post-operative monitoring of tissue transfers: Advantages using contrast enhanced ultrasound (CEUS) and contrast enhanced MRI (ceMRI) with dynamic perfusion analysis? Clin Hemorheol Microcirc 2011: in press

281 ESUR guidelines on contrast media. http://wwwesurorg/Contrastmedia510html\#c270 [accessed June 2011]

282 Thomsen HS. ESUR guideline: gadolinium-based contrast media and nephrogenic systemic fibrosis. Eur Radiol 2007; 17: 2692-2696

Reference No. 5 was modified on September 9, 2011 with respect to the efirst publication. 